\title{
Multiscale Signal-to-Noise Thresholding
}

\author{
W. Freeden, V. Michel, M. Stenger
University of Kaiserslautern
Geomathematics Group
67653 Kaiserslautern
P.O. Box 3049
Germany

$\begin{array}{ll}\text { phone: } & ++49631 \text { 205-2852 } \\ \text { fax: } & ++49631 \text { 29081 } \\ \text { email: } & \text { freeden@mathematik.uni-kl.de } \\ & \text { michel@mathematik.uni-kl.de } \\ & \text { mstenger@mathematik.uni-kl.de } \\ \text { www: } & \text { http://www.mathematik.uni-kl.de/ wwwgeo }\end{array}$

\begin{abstract}
The basic idea behind selective multiscale reconstruction of functions from error-affected data is outlined on the sphere. The selective reconstruction mechanism is based on the premise that multiscale approximation can be well-represented in terms of only a relatively small number of expansion coefficients at various resolution levels. An attempt is made within a tree algorithm (pyramid scheme) to remove the noise component from each scale coefficient using a priori statistical information (provided by an error covariance kernel of a Gaussian, stationary stochastic model).
\end{abstract}

Correspondence to W. Freeden 


\section{Introduction}

While usual Fourier methods in terms of spherical harmonics are very successful at picking out frequencies from a signal, they are utterly incapable of dealing properly with a signal that is changing over space. This fact has been well-known for years. To improve the applicability of Fourier analysis, various methods such as 'windowed Fourier transform' have been developed to adapt the usual Fourier procedure to allow analysis of the frequency content of a signal at each position (cf. W. FrEeden et al. (1998), W. FreEDEN, V. Michel (1999)). However, the amount of localization in space and in frequency is not completely satisfactory. With spherical wavelets, the amount of localization in space and in frequency is automatically adapted, in that only a narrow space-window is needed to examine high-frequency content, but a wide space-window is allowed when investigating low-frequency phenomena.

In physical geodesy the signals mostly are 'output functions' of invariant pseudodifferential operators (such as the gravity anomaly operator, the Stokes' operator, the 'upward and downward continuation' operators, etc). Thus, good space-frequency localization becomes available with spherical wavelets reflecting the rotational symmetry of these operators. This means that those kernel functions are appropriate to examine features of the signal of any size by 'spherical cap windowing'. In other words, the capability of multiresolution analysis is guaranteed by a 'rotation invariant zoom-in, zoom-out' property. The basic framework of this approach has been provided by the spherical wavelet theory developed by the Geomathematics Group at the University of Kaiserslautern during the last years. A generalization to the georelevant harmonic case and its application to spaceborne data has been given by W. FREEDEN (1999). Multiscale developments for the gravimetry problem have been presented by V. Michel (1999).

A signal is a family of measurements, today typically obtained electronically. These quantities could be gravity anomalies, potential values, derivatives at the earth's surface or at a satellite orbit, etc. In signal processing, the interest lies in reconstructing the signal with only minimal loss of receipt. However, signals are typically contaminated by random noise, and an important part of signal processing is accounting for this noise. In consequence, a particular emphasis lies on denoising, i.e. extracting the 'true' signal from the noisy version actually observed. This endeavor is precisely the goal in statistical function estimation. Here, the interest is to 'smooth' the noisy 
data in order to obtain an estimate of the underlying function. In Euclidean theory of wavelets signal processors now have new, fast tools at their disposal that are well-suited for denoising signals, not only those with smooth, wellbehaved natures, but also those originals with strong irregularities (for a survey the reader is e.g. referred to T.R. OGDEN (1997) and the references therein). In fact, the advantages of Euclidean wavelets translate directly over to statistical data analysis. One of the key advantages that Euclidean wavelets have in signal processing are the associated fast algorithms.

The objective of this article is to discuss geodetically relevant spherical wavelets with an eye toward data analysis, giving only the mathematics necessary for a good understanding of how spherical wavelets work in denoising. First the basic ideas of spectral denoising in terms of spherical harmonics are recapitulated in their simplest framework of a Gaussian and stationary stochastic model. With the basic introduction of spherical multiscale approximation, selective thresholding within a pyramid scheme of recursive decomposition is presented. This approach is strongly influenced by the concept of sparse wavelet representation of functions in Euclidean spaces (cf. J.B. Weaver et al. (1991), D.L. Donoho, I.M. Johnstone $(1994,1995))$. The thresholding scheme is designed to distinguish between expansion coefficients that belong to the reconstruction, corresponding to 'true' coefficients which contribute significant signal, and those that do not belong to the reconstruction, corresponding to negligible coefficients. In doing so, the signal is 'projected' onto an approximation space for some small scale (representing the smooth components of the data) and then coefficients at higher resolution are thresholded so that the noise is suppressed but the fine-scale details are included. Finally, some examples are given for multiscale denoising a function involved in an ill-posed problem of physical geodesy (such as the determination of the anomalous potential from gravity anomalies via Stokes' equation and the calculation of the gravitational potential from second order radial derivatives at satellite height via the satellite gradiometry equation).

\section{Preliminaries}

$\mathbb{N}$ denotes the set of all positive integers, and $\mathbb{N}_{0}=\mathbb{N} \cup\{0\}$. As usual, $\mathbb{R}^{3}$ denotes three-dimensional Euclidean space. For all elements $x \in \mathbb{R}^{3}$, 
$x=\left(x_{1}, x_{2}, x_{3}\right)^{T}$, different from the origin, we have

$$
x=r \xi, \quad r=|x|=\sqrt{x_{1}^{2}+x_{2}^{2}+x_{3}^{2}},
$$

where $\xi=\left(\xi_{1}, \xi_{2}, \xi_{3}\right)^{T}$ is the uniquely determined directional unit vector of $x$. The unit sphere in $\mathbb{R}^{3}$ is denoted by $\Omega$. If the vectors $\varepsilon^{1}, \varepsilon^{2}, \varepsilon^{3}$ form the canonical orthonormal basis in $\mathbb{R}^{3}$, the points $\xi \in \Omega$ may be represented in polar coordinates by

$$
\begin{aligned}
\xi & =t \varepsilon^{3}+\sqrt{1-t^{2}}\left(\cos \varphi \varepsilon^{1}+\sin \varphi \varepsilon^{2}\right) \\
t & =\cos \vartheta, \vartheta \in[0, \pi], \varphi \in[0,2 \pi) .
\end{aligned}
$$

\subsection{Spherical Harmonics}

The spherical harmonics $Y_{n}$ of degree $n$ are defined as the everywhere on $\Omega$ infinitely differentiable eigenfunctions of the Beltrami operator $\Delta^{*}$ corresponding to the eigenvalues $\left(\Delta^{*}\right)^{\wedge}(n)=-n(n+1), n=0,1, \ldots$, where the Beltrami-operator is the spherical part of the Laplace operator in $\mathbb{R}^{3}$. As it is well-known, the functions $H_{n}: \mathbb{R}^{3} \rightarrow \mathbb{R}$ defined by $H_{n}(x)=r^{n} Y_{n}(\xi), x=r \xi$, are homogeneous polynomials in rectangular coordinates which satisfy the Laplace equation $\Delta_{x} H_{n}(x)=0, x \in \mathbb{R}^{3}$. Conversely, every homogeneous harmonic polynomial of degree $n$ when restricted to $\Omega$ is a spherical harmonic of degree $n$. The Legendre polynomials $P_{n}:[-1,+1] \rightarrow[-1,+1]$ are the only everywhere on $[-1,+1]$ infinitely differentiable eigenfunctions of the Legendre operator $\left(1-t^{2}\right)(d / d t)^{2}-2 t(d / d t)$, which satisfy $P_{n}(1)=1$. Apart from a multiplicative constant, the 'Legendre function' $P_{n}\left(\varepsilon^{3} \cdot\right): \Omega \rightarrow[-1,+1]$, $\xi \mapsto P_{n}\left(\varepsilon^{3} \cdot \xi\right), \xi \in \Omega$, is the only spherical harmonic of degree $n$ which is invariant under orthogonal transformations leaving $\varepsilon^{3}$ fixed. The linear space $\mathrm{Harm}_{n}$ of all spherical harmonics of degree $n$ is of dimension $\operatorname{dim}\left(\operatorname{Harm}_{n}\right)=2 n+1$. Thus, there exist $2 n+1$ linearly independent spherical harmonics $Y_{n, 1}, \ldots, Y_{n, 2 n+1}$ in $\operatorname{Harm}_{n}$. Throughout the remainder of this paper we assume this system to be orthonormal in the sense of the $\mathcal{L}^{2}(\Omega)$-inner product

$$
\left(Y_{n, j}, Y_{m, k}\right)_{\mathcal{L}^{2}(\Omega)}=\int_{\Omega} Y_{n, j}(\eta) Y_{m, k}(\eta) d \omega(\eta)=\delta_{n, m} \delta_{j, k}
$$

( $d \omega$ denotes the surface element). An outstanding result of the theory of spherical harmonics is the addition theorem

$$
\sum_{k=1}^{2 n+1} Y_{n, k}(\xi) Y_{n, k}(\eta)=\frac{2 n+1}{4 \pi} P_{n}(\xi \cdot \eta), \quad(\xi, \eta) \in \Omega \times \Omega .
$$


The close connection between the orthogonal invariance and the addition theorem is established by the Funk-Hecke formula

$$
\int_{\Omega} H(\xi \cdot \eta) P_{n}(\zeta \cdot \eta) d \omega(\eta)=\left(H(\cdot \xi), P_{n}(\cdot \zeta)\right)_{\mathcal{L}^{2}(\Omega)}=H^{\wedge}(n) P_{n}(\xi \cdot \zeta),
$$

$H \in \mathcal{L}^{1}[-1,+1], \xi, \zeta \in \Omega$, where the Legende transform $H^{\wedge}(n)$ is given by

$$
H^{\wedge}(n)=2 \pi \int_{-1}^{+1} H(t) P_{n}(t) d t, \quad n=0,1, \ldots .
$$

The sequence $\left\{H^{\wedge}(n)\right\}_{n \in \mathbb{N}_{0}}$ is called the symbol of $H$. For more details about the theory of spherical harmonics the reader is referred, for example, to C. MÜLler (1966) and W. FrEEDEN et al. (1998).

We let $\Xi(\Omega)$ stand either for the space $C(\Omega)$ or $\mathcal{L}^{p}(\Omega), 1 \leq p<\infty$, (with the corresponding norm $\left.\|\cdot\|_{\Xi(\Omega)}\right)$. In what follows we are mainly interested, however, in results for the Hilbert space $\left(\mathcal{L}^{2}(\Omega),(\cdot, \cdot)_{\mathcal{L}^{2}(\Omega)}\right)$. Any function of the form $H_{\xi}: \Omega \rightarrow \mathbb{R}, \eta \mapsto H_{\xi}(\eta)=H(\xi \cdot \eta), \eta \in \Omega$, is called a $\xi$-zonal function on $\Omega$. Zonal functions are constant on the sets of all $\eta \in \Omega$, with $\xi \cdot \eta=h, h \in[-1,+1]$. The set of all $\xi$-zonal functions is isomorphic to the set of functions $H:[-1,+1] \rightarrow \mathbb{R}$. This gives rise to interpret the spaces $C[-1,+1]$ and $\mathcal{L}^{2}[-1,+1]$ with norms defined correspondingly as subspaces of $C(\Omega)$ and $\mathcal{L}^{2}(\Omega)$. Analogously, we let $\Xi[-1,+1]$ stand either for the space $C[-1,+1]$ or $\mathcal{L}^{p}[-1,+1], 1 \leq p<\infty$ (with the corresponding norm $\left.\|\cdot\|_{\Xi[-1,+1]}\right)$. In particular,

$\|H\|_{\mathcal{L}^{2}[-1,+1]}=\left(2 \pi \int_{-1}^{+1}|H(t)|^{2} d t\right)^{1 / 2}=\left\|H\left(\varepsilon^{3} \cdot\right)\right\|_{\mathcal{L}^{2}(\Omega)}, \quad H \in \mathcal{L}^{2}[-1,+1]$.

\subsection{Spectral Approximation}

The spherical Fourier transform $H \mapsto(F T)(H), H \in \mathcal{L}^{2}(\Omega)$, is given by

$$
((F T)(H))(n, k)=H^{\wedge}(n, k)=\left(H, Y_{n, k}\right)_{\mathcal{L}^{2}(\Omega)} .
$$


$F T$ forms a mapping from $\mathcal{L}^{2}(\Omega)$ onto the space $l^{2}(\mathcal{N})$ of all sequences $\left\{H_{n, k}\right\}$ satisfying

$$
\sum_{(n, k) \in \mathcal{N}} H_{n, k}^{2}=\sum_{n=0}^{\infty} \sum_{k=1}^{2 n+1} H_{n, k}^{2}<\infty
$$

where

$$
\mathcal{N}=\{(n, k) \mid n=0,1, \ldots ; k=1, \ldots, 2 n+1\} .
$$

For $\left\{H_{n, k}\right\} \in l^{2}(\mathcal{N})$ we define the mapping $(F T)^{-1}: l^{2}(\mathcal{N}) \rightarrow \mathcal{L}^{2}(\Omega)$ by

$$
(F T)^{-1}\left(H_{n, j}\right)=\sum_{n=0}^{\infty} \sum_{k=1}^{2 n+1} H_{n, k} Y_{n, k} .
$$

Then $(F T)^{-1}(F T)=I_{\mathcal{L}^{2}(\Omega)}$ and $(F T)(F T)^{-1}=I_{l^{2}(\mathcal{N})}(I$ is the identity operator). Moreover, it should be noted that for $G, H \in \mathcal{L}^{2}(\Omega)$ the relation

$$
\lim _{N \rightarrow \infty}\left\|G-\sum_{n=0}^{N} \sum_{k=1}^{2 n+1} H^{\wedge}(n, k) Y_{n, k}\right\|_{\mathcal{L}^{2}(\Omega)}=0
$$

implies $G=H$ almost everywhere on the unit sphere $\Omega$. The series

$$
\sum_{n=0}^{\infty} \sum_{k=1}^{2 n+1} F^{\wedge}(n, k) Y_{n, k}
$$

is called the spherical Fourier expansion of $F$ (with Fourier coefficients $\left.F^{\wedge}(n, k), n=0,1, \ldots ; k=1, \ldots, 2 n+1\right)$. For all $F \in \mathcal{L}^{2}(\Omega)$ the property

$$
\lim _{N \rightarrow \infty}\left\|F-\sum_{n=0}^{N} \sum_{k=1}^{2 n+1} F^{\wedge}(n, k) Y_{n, k}\right\|_{\mathcal{L}^{2}(\Omega)}=0
$$

is equivalent to the Parseval identity

$$
\|F\|_{\mathcal{L}^{2}(\Omega)}^{2}=(F, F)_{\mathcal{L}^{2}(\Omega)}=\sum_{n=0}^{\infty} \sum_{k=1}^{2 n+1}\left(F^{\wedge}(n, k)\right)^{2} .
$$

The recovery of a function $F \in \mathcal{L}^{2}(\Omega)$ by its Fourier expansion (in the sense of $\|\cdot\|_{\mathcal{L}^{2}(\Omega)}$ ) is equivalent to the following conditions (see, for example W. FrEEDEN et al. (1998)): 
(i) (closure). The system $\left\{Y_{n, k}\right\}_{n=0,1, \ldots ; k=1, \ldots, 2 n+1}$ is closed in $\mathcal{L}^{2}(\Omega)$, i.e. for any number $\varepsilon>0$ and any function $F \in \mathcal{L}^{2}(\Omega)$ there exists a linear combination $Z=\sum_{n=0}^{N} \sum_{k=1}^{2 n+1} d_{n, k} Y_{n, k}$ such that $\|F-Z\|_{\mathcal{L}^{2}(\Omega)} \leq \varepsilon$.

(ii) (completeness). The system $\left\{Y_{n, k}\right\}_{n=0,1, \ldots ; k=1, \ldots, 2 n+1}$ is complete in $\mathcal{L}^{2}(\Omega)$, i.e. $F \in \mathcal{L}^{2}(\Omega)$ with $F^{\wedge}(n, k)=0$ for $n=0,1, \ldots ; k=$ $1, \ldots, 2 n+1$ implies $F=0$.

(iii) The system $\left\{Y_{n, k}\right\}_{n=0,1, \ldots ; k=1, \ldots, 2 n+1}$ is a Hilbert basis of $\mathcal{L}^{2}(\Omega)$, i.e.

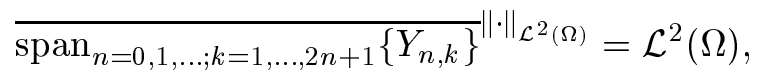

where 'span' means the set of all finite linear combinations.

\subsection{Multiscale Approximation}

Assume that $H \in \mathcal{L}^{2}[-1,+1]$ and $F \in \mathcal{L}^{2}(\Omega)$. Then the convolution of $H$ against $F$ is defined by

$$
H * F=\int_{\Omega} H(\cdot \eta) F(\eta) d \omega(\eta) .
$$

Two important properties of spherical convolutions should be listed: (i) If $F \in \mathcal{L}^{2}(\Omega)$ and $H \in \mathcal{L}^{2}[-1,+1]$, then $H * F$ is of class $\mathcal{L}^{2}(\Omega)$. (ii) If $H_{1}, H_{2} \in \mathcal{L}^{2}[-1,+1]$, then the convolution of $H_{1}, H_{2}$ is of class $C[-1,+1]$, and we have

$$
\left(H_{1} * H_{2}\right)(\xi \cdot \zeta)=\int_{\Omega} H_{1}(\xi \cdot \eta) H_{2}(\zeta \cdot \eta) d \omega(\eta)
$$

and

$$
\left(H_{1} * H_{2}\right)^{\wedge}(n)=H_{1}^{\wedge}(n) H_{2}^{\wedge}(n), \quad n \in \mathbb{N}_{0} .
$$

We usually write $H^{(2)}=H * H$ to indicate the convolution of $H \in \mathcal{L}^{2}[-1,+1]$ with itself. $H^{(2)}$ is said to be the second iterated kernel of $H$. More general, $H^{(p)}=H^{(p-1)} * H$ for $p=2,3, \ldots$ and $H^{(1)}=H$. Obviously,

$$
\left(H^{(p)}\right)^{\wedge}(n)=\left(H^{\wedge}(n)\right)^{p}, \quad n \in \mathbb{N}_{0}, \quad p \in \mathbb{N} .
$$

Next we consider a strict monotonically decreasing sequence $\left\{\rho_{j}\right\}_{j \in \mathbb{Z}}$ of real numbers satisfying

$$
\lim _{j \rightarrow \infty} \rho_{j}=0 \text { and } \lim _{j \rightarrow-\infty} \rho_{j}=\infty
$$


(for example, $\rho_{j}=2^{-j}, j \in \mathbb{Z}$ ). The sequence $\left\{\rho_{j}\right\}_{j \in \mathbb{Z}}$ can be understood as a subdivision of the 'scale interval' $(0, \infty)$ into a countable, strict monotonically decreasing sequence.

Let $\left\{\Phi_{\rho_{j}}\right\}_{j \in \mathbb{Z}}$ be a subfamily of $\mathcal{L}^{2}[-1,+1]$ satisfying the condition

$$
\Phi_{\rho_{j}}^{\wedge}(0)=1
$$

for all $j \in \mathbb{Z}$. Then, the family $\left\{I_{\rho_{j}}\right\}_{j \in \mathbb{Z}}$ of operators $I_{\rho_{j}}$, defined by

$$
I_{\rho_{j}}(F)=\Phi_{\rho_{j}} * F, \quad F \in \mathcal{L}^{2}(\Omega),
$$

is called a singular integral in $\mathcal{L}^{2}(\Omega) .\left\{\Phi_{\rho_{j}}\right\}_{j \in \mathbb{Z}}$ is called kernel of the singular integral.

If $\left\{\Phi_{\rho_{j}}\right\}_{j \in \mathbb{Z}}$ is a kernel of a singular integral satisfying the conditions

(i) for all $n \in \mathbb{N}_{0} \lim _{j \rightarrow \infty} \Phi_{\rho_{j}}^{\wedge}(n)=1$ and $\lim _{j \rightarrow-\infty} \Phi_{\rho_{j}}^{\wedge}(n)=0$,

(ii) there exists $C>0$ such that $\sup _{n \in \mathbb{N}_{0}}\left|\Phi_{\rho_{j}}^{\wedge}(n)\right| \leq C$ for all $j \in \mathbb{Z}$,

then the corresponding singular integral $\left\{I_{\rho_{j}}\right\}_{j \in \mathbb{Z}}$ is called approximate identity.

It is known (see e.g. W. FreEDEN et al. (1998)) that

$$
\begin{aligned}
& \lim _{j \rightarrow \infty}\left\|I_{\rho_{j}}(F)-F\right\|_{\mathcal{L}^{2}(\Omega)} \\
& =\lim _{j \rightarrow \infty}\left(\sum_{n=0}^{\infty} \sum_{k=1}^{2 n+1}\left(F^{\wedge}(n, k)\right)^{2}\left(1-\Phi_{\rho_{j}}^{\wedge}(n)\right)^{2}\right)^{1 / 2} \\
& =0,
\end{aligned}
$$

provided that $\left\{I_{\rho_{j}}\right\}_{j \in \mathbb{Z}}$ is an approximate identity.

Assume that the kernel $\left\{\Phi_{\rho_{j}}\right\}_{j \in \mathbb{Z}}$ is non-negative (i.e. $\Phi_{\rho_{j}}(t) \geq 0$ almost everywhere on the interval $[-1,+1]$ for all $j \in \mathbb{Z})$. Then the following properties are equivalent (cf. W. FreEden, K. Hesse (2000)):

(i) $\left\{I_{\rho_{j}}\right\}_{j \in \mathbb{Z}}$ is an approximate identity in $\mathcal{L}^{2}(\Omega)$,

(ii) $\lim _{j \rightarrow \infty} \Phi_{\rho_{j}}^{\wedge}(n)=1$ for all $n \in \mathbb{N}_{0}$,

(iii) $\lim _{j \rightarrow \infty} \Phi_{\rho_{j}}^{\wedge}(1)=1$, 
(iv) $\lim _{j \rightarrow \infty} \int_{-1}^{\delta} \Phi_{\rho_{j}}(t) d t=0$ for all $\delta \in(-1,+1)$ (localization property).

Assume that $\left\{I_{\rho_{j}}\right\}_{j \in \mathbb{Z}}$ is an approximate identity in $\mathcal{L}^{2}(\Omega)$. Then it follows that $\left\{I_{\rho_{j}}^{(q)}\right\}, q \in \mathbb{N}$, defined by

$$
I_{\rho_{j}}^{(q)}(F)=\Phi_{\rho_{j}}^{(q)} * F, \quad F \in \mathcal{L}^{2}(\Omega),
$$

is an approximate identity in $\mathcal{L}^{2}(\Omega)$ (observe that $\left(\Phi_{\rho_{j}}^{(q)}\right)^{\wedge}(n)=\left(\left(\Phi_{\rho_{j}}\right)^{\wedge}(n)\right)^{q}$ for all $n \in \mathbb{N}_{0}$ such that $\lim _{j \rightarrow \infty}\left(\Phi_{\rho_{j}}^{(q)}\right)^{\wedge}(n)=1$ for all $\left.n \in \mathbb{N}_{0}\right)$.

Our results lead us to the following statement: Assume that $\left\{\Phi_{\rho_{j}}\right\}_{j \in \mathbb{Z}}$ is a kernel constituting an approximate identity in $\mathcal{L}^{2}(\Omega)$. Then the limit relation

$$
\begin{aligned}
& \lim _{j \rightarrow \infty}\left\|\int_{\Omega} \Phi_{\rho_{j}}^{(2)}(\cdot \eta) F(\eta) d \omega(\eta)-F\right\|_{\mathcal{L}^{2}(\Omega)} \\
& =\lim _{j \rightarrow \infty}\left\|\int_{\Omega} \int_{\Omega} \Phi_{\rho_{j}}(\eta \cdot \zeta) F(\zeta) d \omega(\zeta) \Phi_{\rho_{j}}(\cdot \eta) d \omega(\eta)-F\right\|_{\mathcal{L}^{2}(\Omega)}=0
\end{aligned}
$$

holds for all $F \in \mathcal{L}^{2}(\Omega)$.

For $J \in \mathbb{Z}$ we set

$$
F_{J}=\Phi_{\rho_{J}}^{(2)} * F=\int_{\Omega} \Phi_{\rho_{J}}^{(2)}(\cdot \eta) F(\eta) d \omega(\eta)
$$

Consider a kernel $\left\{\Phi_{\rho_{j}}\right\}_{j \in \mathbb{Z}}$ constituting an approximate identity in $\mathcal{L}^{2}(\Omega)$. Assume that $F$ is of class $\mathcal{L}^{2}(\Omega)$. Then a simple calculation shows us that for all $N \in \mathbb{N}$ and $J \in \mathbb{Z}$,

$$
\begin{aligned}
\int_{\Omega} \Phi_{\rho_{J+N}}^{(2)}(\cdot \eta) F(\eta) d \omega(\eta) & =\int_{\Omega} \Phi_{\rho_{J}}^{(2)}(\cdot \eta) F(\eta) d \omega(\eta) \\
& +\sum_{j=J}^{J+N-1} \int_{\Omega} \Psi_{\rho_{j}}^{(2)}(\cdot \eta) F(\eta) d \omega(\eta)
\end{aligned}
$$

where we have used the abbreviation

$$
\Psi_{\rho_{j}}^{(2)}(\xi \cdot \eta)=\Phi_{\rho_{j+1}}^{(2)}(\xi \cdot \eta)-\Phi_{\rho_{j}}^{(2)}(\xi \cdot \eta)
$$


$j \in \mathbb{Z},(\xi, \eta) \in \Omega \times \Omega$. Hence, letting $N$ tend to infinity we get the following multiscale reconstruction formula

$$
F=F_{J}+\sum_{j=J}^{\infty} \int_{\Omega} \Psi_{\rho_{j}}^{(2)}(\cdot \eta) F(\eta) d \omega(\eta)
$$

for every $J \in \mathbb{Z}$ (in the sense of $\mathcal{L}^{2}(\Omega)$ ).

Moreover, we find

$$
\int_{\Omega} \Phi_{\rho_{J}}^{(2)}(\cdot \eta) F(\eta) d \omega(\eta)=F_{J-N}+\sum_{j=J-N}^{J-1} \int_{\Omega} \Psi_{\rho_{j}}^{(2)}(\cdot \eta) F(\eta) d \omega(\eta),
$$

hence,

$$
\int_{\Omega} \Phi_{\rho_{J}}^{(2)}(\cdot \eta) F(\eta) d \omega(\eta)=\sum_{j=-\infty}^{J-1} \int_{\Omega} \Psi_{\rho_{j}}^{(2)}(\cdot \eta) F(\eta) d \omega(\eta) .
$$

Combining (31) and (33) we finally obtain the following multiscale representation of $F \in \mathcal{L}^{2}(\Omega)$ in the sense of $\|\cdot\|_{\mathcal{L}^{2}(\Omega)}$

$$
F=\sum_{j=-\infty}^{\infty} \int_{\Omega} \Psi_{\rho_{j}}^{(2)}(\cdot \eta) F(\eta) d \omega(\eta)
$$

provided that the so-called 'scaling function' $\left\{\Phi_{\rho_{j}}\right\}_{j \in \mathbb{Z}}$ forms an approximate identity in $\mathcal{L}^{2}(\Omega)$ and the 'wavelet' $\left\{\Psi_{\rho_{j}}\right\}_{j \in \mathbb{Z}}$ satisfies the difference equation (30).

The class $\mathcal{V}_{\rho_{j}}$ of all functions $P \in \mathcal{L}^{2}(\Omega)$ of the form

$$
P=\Phi_{\rho_{j}}^{(2)} * F, \quad F \in \mathcal{L}^{2}(\Omega),
$$

is called the scale space of level $j$ (with respect to the scaling function $\left.\left\{\Phi_{\rho_{j}}\right\}_{j \in \mathbb{Z}}\right)$, whereas the class $\mathcal{W}_{\rho_{j}}$ of all functions $Q \in \mathcal{L}^{2}(\Omega)$ of the representation

$$
Q=\Psi_{\rho_{j}}^{(2)} * F, \quad F \in \mathcal{L}^{2}(\Omega),
$$

is called the detail space of level $j$ (with respect to the scaling function $\left.\left\{\Phi_{\rho_{j}}\right\}_{j \in \mathbb{Z}}\right)$. It is easily seen from (29) that

$$
\mathcal{V}_{\rho_{j+1}}=\mathcal{V}_{\rho_{j}}+\mathcal{W}_{\rho_{j}}
$$

for all $j \in \mathbb{Z}$. But it should be remarked that the sum (37) generally is neither direct nor orthogonal (note that an orthogonal decomposition is given by 
the Shannon scaling function). The equation (37) can be interpreted in the following way: The set $\mathcal{V}_{\rho_{j}}$ contains a filtered ('smoothed') version of a function belonging to $\mathcal{L}^{2}(\Omega)$. The lower the scale, the stronger the intensity of smoothing. By adding 'details' contained in the detail space $\mathcal{W}_{\rho_{j}}$ the space $\mathcal{V}_{\rho_{j+1}}$ is created, which consists of a filtered ('smoothed') version at resolution $j+1$ (cf. W. FreEden et al. (1998), W. FreEden (1999)).

Finally, for all $n=0,1, \ldots ; k=1, \ldots, 2 n+1$, we obtain

$$
F^{\wedge}(n, k)=\sum_{j=-\infty}^{\infty} F^{\wedge}(n, k)\left(\Psi_{\rho_{j}}^{(2)}\right)^{\wedge}(n) .
$$

But this means that

$$
\sum_{j=-\infty}^{+\infty}\left(\Psi_{\rho_{j}}^{(2)}\right)^{\wedge}(n)=1
$$

for $n=0,1, \ldots$ By construction we are therefore led to a partition of unity as follows

$$
\begin{aligned}
\sum_{j=-\infty}^{+\infty}\left(\Psi_{\rho_{j}}^{\wedge}(n)\right)^{2} & =\left(\sum_{j=-\infty}^{J-1}+\sum_{j=J}^{\infty}\right)\left(\Psi_{\rho_{j}}^{\wedge}(n)\right)^{2} \\
& =\left(\Phi_{\rho_{J}}^{\wedge}(n)\right)^{2}+\sum_{j=J}^{\infty}\left(\Psi_{\rho_{j}}^{\wedge}(n)\right)^{2} \\
& =\left(\Phi_{\rho_{J}}^{\wedge}(n)\right)^{2}+\sum_{j=J}^{\infty}\left(\left(\Phi_{\rho_{j+1}}^{\wedge}(n)\right)^{2}-\left(\Phi_{\rho_{j}}^{\wedge}(n)\right)^{2}\right) \\
& =1
\end{aligned}
$$

for $n \in \mathbb{N}_{0}$.

Remark. Before we continue with some examples, the above definition of 'difference wavelets' should be motivated in comparison to the usual concept of Euclidean wavelet theory. For that purpose we mention that the Euclidean interpretation of $\Phi_{\rho_{j+1}}^{(2)}-\Phi_{\rho_{j}}^{(2)}$ is based on a (scale continuous) function $\breve{\Psi}_{\rho}$ (which is specified in more detail in the standard literature of Euclidean wavelet theory, see, for example, C.K. CHui (1992), A.K. Louis et al. (1994)) such that

$$
\Phi_{\rho_{j+1}}^{(2)}-\Phi_{\rho_{j}}^{(2)}=\int_{\rho_{j}}^{\rho_{j+1}} \breve{\Psi}_{\rho}^{(2)} \frac{d \rho}{\rho} .
$$


Now the relation between the interpretation practised in Euclidean theory and our approach developed above is given by

$$
\Phi_{\rho_{j+1}}^{(2)}-\Phi_{\rho_{j}}^{(2)}=\Psi_{\rho_{j}}^{(2)} \approx-\breve{\Psi}_{\rho_{j+1}}^{(2)} \frac{\rho_{j+1}-\rho_{j}}{\rho_{j+1}}
$$

Particularly, in dyadic scales, the last formula reads

$$
\begin{aligned}
\Phi_{2^{-(j+1)}}^{(2)}-\Phi_{2^{-j}}^{(2)} & =\Psi_{2^{-j}}^{(2)} \\
& \approx-\breve{\Psi}_{2^{-(j+1)}}^{(2)} \frac{2^{-(j+1)}-2^{-j}}{2^{-(j+1)}} \\
& =\breve{\Psi}_{2^{-(j+1)}}^{(2)} .
\end{aligned}
$$

\subsection{Examples}

Singular integrals on the sphere are of basic interest in geomathematical applications. We essentially distinguish two types, namely bandlimited and non-bandlimited singular integrals.

\subsubsection{Bandlimited Singular Integrals}

Shannon Singular Integral. The family $\left\{\Phi_{\rho_{j}}\right\}_{j \in \mathbb{Z}}$ is defined by

$$
\Phi_{\rho_{j}}^{\wedge}(n)=\left\{\begin{array}{lll}
1 & \text { for } & n \in\left[0, \rho_{j}^{-1}\right) \\
0 & \text { for } & n \in\left[\rho_{j}^{-1}, \infty\right)
\end{array}\right.
$$

with a strict monotonically decreasing sequence of integers $\left\{\rho_{j}\right\}_{j \in \mathbb{Z}}$ satisfying

$$
\lim _{j \rightarrow-\infty} \rho_{j}=\infty \quad \text { and } \quad \lim _{j \rightarrow \infty} \rho_{j}=0
$$

(for example: $\rho_{j}=2^{-j}$ ).

Smoothed Shannon Singular Integral. The family $\left\{\Phi_{\rho_{j}}\right\}_{j \in \mathbb{Z}}$ is given by

$$
\Phi_{\rho_{j}}^{\wedge}(n)=\left\{\begin{array}{ccc}
1 & \text { for } & n \in\left[0, \sigma_{j}^{-1}\right) \\
\tau_{j}(n) & \text { for } & n \in\left[\sigma_{j}^{-1}, \rho_{j}^{-1}\right) \\
0 & \text { for } & n \in\left[\rho_{j}^{-1}, \infty\right) .
\end{array}\right.
$$


where $\left\{\rho_{j}\right\}_{j \in \mathbb{Z}}$ is defined as in the Shannon case and $\left\{\sigma_{j}\right\}_{j \in \mathbb{Z}}$ is a strict monotonically decreasing sequence of integers satisfying

$$
\begin{gathered}
\lim _{j \rightarrow-\infty} \sigma_{j}=\infty \text { and } \lim _{j \rightarrow \infty} \sigma_{j}=0, \\
\sigma_{j}>\rho_{j},
\end{gathered}
$$

and $\tau_{j}$ is a strict monotonically decreasing continuous function of class $C\left[\sigma_{j}^{-1}, \rho_{j}^{-1}\right], j \geq 0$ such that

$$
\tau_{j}\left(\sigma_{j}^{-1}\right)=1, \tau_{j}\left(\rho_{j}^{-1}\right)=0,
$$

for example $\tau_{j}(t)=2-2^{-j} t$ with $\rho_{j}=2^{-j-1}$ and $\sigma_{j}=2^{-j}$.

\subsubsection{Non-bandlimited Singular Integrals}

Abel-Poisson Singular Integral. The family $\left\{\Phi_{\rho_{j}}\right\}_{j \in \mathbb{Z}}$ is given by

$$
\Phi_{\rho_{j}}^{\wedge}(n)=e^{-n \rho_{j}}, \quad n \in \mathbb{N}_{0}, j \in \mathbb{Z} .
$$

Tikhonov Singular Integral. The family $\left\{\Phi_{\rho_{j}}\right\}_{j \in \mathbb{Z}}$ is given by

$$
\Phi_{\rho_{j}}^{\wedge}(n)=\frac{\sigma_{n}^{2}}{\sigma_{n}^{2}+\rho_{j}^{2}}, \quad n \in \mathbb{N}_{0}, j \in \mathbb{Z},
$$

where $\left\{\sigma_{n}\right\}_{n \in \mathbb{N}_{0}}$ is a summable sequence, i.e. $\sigma_{n} \neq 0$ for all $n \in \mathbb{N}_{0}$ and

$$
\sum_{n=0}^{\infty} \frac{2 n+1}{4 \pi} \sigma_{n}^{2}<\infty
$$

Locally Supported Singular Integral. The family $\left\{L_{\rho_{j}}^{(k)}\right\}_{j \in \mathbb{Z}}$ is given by

$$
\left(L_{\rho_{j}}^{(k)}\right)^{\wedge}(n)=2 \pi \int_{-1}^{+1} L_{\rho_{j}}^{(k)}(t) P_{n}(t) d t, \quad n \in \mathbb{N}_{0}
$$

with

$$
L_{\rho_{j}}^{(k)}(t)=\left\{\begin{array}{cl}
0 & \text { for }-1 \leq t \leq 1-\rho_{j} \\
\frac{1}{2 \pi} \frac{k+1}{\rho_{j}^{k+1}}\left(t-1+\rho_{j}\right)^{k} & \text { for } 1-\rho_{j}<t \leq 1 .
\end{array}\right.
$$

For the case $k=0$ this example is known as the Haar singular integral. From J. CUI et al. (1992) we know that $\left(L_{\rho_{j}}^{(k)}\right)^{\wedge}(n)=O\left(n^{-(3 / 2)-k}\right)$ for $n \rightarrow \infty$. 


\section{Spectral Signal-to-Noise Response}

Geoscientists mostly think of their measurements (after possible linearization) as a linear operator on an 'input signal' $F$ producing an 'output signal' $G$

$$
\Lambda F=G,
$$

where $\Lambda$ is an operator mapping the space $\mathcal{L}^{2}(\Omega)$ into itself.

Usually $\Lambda$ is an (rotation-invariant) operator such that

$$
\Lambda Y_{n, k}=\Lambda^{\wedge}(n) Y_{n, k}, \quad n=0,1, \ldots ; k=1, \ldots, 2 n+1,
$$

where the so-called symbol $\left\{\Lambda^{\wedge}(n)\right\}_{n \in \mathbb{N}_{0}}$ is the sequence of the real numbers $\Lambda^{\wedge}(n)$. Different linear operators $\Lambda$, of course, are characterized by different sequences $\left\{\Lambda^{\wedge}(n)\right\}_{n \in \mathbb{N}_{0}}$ (for more details see P. MeIssL (1971), S.L. SVENSSON (1983), W. FreEDEn et al. (1998)). The 'amplitude spectrum' $\left\{G^{\wedge}(n, k)\right\}_{(n, k) \in \mathcal{N}}$ of the response of $\Lambda$ is described in terms of the amplitude spectrum of functions (signals) by a simple multiplication by the 'transfer' $\Lambda^{\wedge}(n)$

\begin{tabular}{|c|c|}
\hline operator & symbol \\
\hline first radial derivative & $n+1$ \\
\hline second radial derivative & $(n+1)(n+2)$ \\
\hline gravity anomaly & $n-1$ \\
\hline Stokes' & $\frac{1}{n-1}, n>1$ \\
\hline single layer potential & $\frac{1}{n+(1 / 2)}$ \\
\hline double layer potential & $-\frac{1}{2 n+1}$ \\
\hline
\end{tabular}

Table 1: Geodetically relevant operators on $\Omega$

\section{$2.1 \quad$ Noise Model}

Thus far only a (deterministic) function model has been discussed. If a comparison of the 'output function' with the actual value were done, dis- 
crepancies would be observed. A mathematical description of these discrepancies has to follow the laws of probability theory in a stochastic model. In physical geodesy, in order to arrive at a spectral error model of sufficient simplicity, the measurement process usually is considered to be Gaussian and stationary. Only if it is Gaussian (normally distributed), its first two statistical moments, i.e. mean value and variance, are sufficient for a complete description. Only if it is stationary (homogeneous and isotropic), the error spectrum can be considered to be rotational invariant and, therefore, representative for almost all scalar observables of physical geodesy (as already mentioned most observables of physical geodesy are characterized by scalar invariant pseudodifferential operators $\Lambda$ )

Usually the observations are not looked upon as a time series (see W.A. Heiskanen, H. Moritz (1967), F. Sansò, R. Rummel (1997), R. RumMEL (1997) and many others), but rather a function $\tilde{G}$ on the sphere $\Omega($ ' for stochastic). According to this approach it is assumed that, we have

$$
\tilde{G}=G+\tilde{\varepsilon},
$$

where $\tilde{\varepsilon}$ is the observation noise. The two stochastical moments have to fulfill

$$
\begin{aligned}
E[\tilde{G}(\xi)] & =G(\xi), \quad \xi \in \Omega, \\
\operatorname{var}[\tilde{G}(\xi)] & =\operatorname{var}[\tilde{\epsilon}(\xi)]=\sigma^{2}, \quad \xi \in \Omega,
\end{aligned}
$$

and

$$
\operatorname{cov}[\tilde{G}(\xi), \tilde{G}(\eta)]=K(\xi \cdot \eta), \quad(\xi, \eta) \in \Omega \times \Omega .
$$

In other words, $\tilde{\epsilon}$ is assumed to be $N\left(0, \sigma^{2}\right)$-distributed, such that

$$
E[\tilde{\varepsilon}(\xi) \cdot \tilde{\varepsilon}(\eta)]=K(\xi \cdot \eta), \quad(\xi, \eta) \in \Omega \times \Omega,
$$

where mathematical arguments lead us to assume that the following conditions are imposed on the symbol $\left\{K^{\wedge}(n)\right\}_{n \in \mathbb{N}_{0}}$ :

(C1) $K^{\wedge}(n) \geq 0$ for all $n \geq 0$,

(C2) $\sum_{n=0}^{\infty} \frac{2 n+1}{4 \pi} K^{\wedge}(n)<\infty$.

Both conditions $(\mathrm{C} 1)$ and $(\mathrm{C} 2)$, indeed, imply the summability of the symbol $\left\{K^{\wedge}(n)\right\}_{n \in \mathbb{N}_{0}}$. 


\subsection{Degree Variances}

As any 'output function' (output signal) can be expanded into an orthogonal series of surface spherical harmonics

$$
\begin{aligned}
\tilde{G}=\widetilde{\Lambda F} & =\sum_{n=0}^{\infty} \sum_{k=1}^{2 n+1} \Lambda^{\wedge}(n) \tilde{F}^{\wedge}(n, k) Y_{n, k} \\
& =\sum_{n=0}^{\infty} \sum_{k=1}^{2 n+1} \tilde{G}^{\wedge}(n, k) Y_{n, k}
\end{aligned}
$$

in the sense of $\|\cdot\|_{\mathcal{L}^{2}(\Omega)}$, we get a spectral representation of the form

$$
\tilde{G}^{\wedge}(n, k)=\Lambda^{\wedge}(n) \tilde{F}^{\wedge}(n, k),
$$

$n=0,1, \ldots ; k=1, \ldots, 2 n+1$.

The signal degree-order variances of $\tilde{G}=\widetilde{\Lambda F}$ are defined by

$$
\begin{aligned}
\operatorname{var}_{n, k}(\widetilde{\Lambda F}) & =\left(\int_{\Omega}(\widetilde{\Lambda F})(\eta) Y_{n, k}(\eta) d \omega(\eta)\right)^{2} \\
& =\left((\widetilde{\Lambda F})^{\wedge}(n, k)\right)^{2} \\
& =\int_{\Omega} \int_{\Omega}(\widetilde{\Lambda F})(\xi)(\widetilde{\Lambda F})(\eta) Y_{n, k}(\xi) Y_{n, k}(\eta) d \omega(\xi) d \omega(\eta) .
\end{aligned}
$$

Correspondingly, the signal degree variances of $\tilde{G}=\widetilde{\Lambda F}$ are given by

$$
\begin{aligned}
\operatorname{var}_{n}(\widetilde{\Lambda F}) & =\sum_{k=1}^{2 n+1} \operatorname{var}_{n, k}(\widetilde{\Lambda F}) \\
& =\sum_{k=1}^{2 n+1}\left(\int_{\Omega}(\widetilde{\Lambda F})(\eta) Y_{n, k}(\eta) d \omega(\eta)\right)^{2} \\
& =\sum_{k=1}^{2 n+1}\left((\widetilde{\Lambda F})^{\wedge}(n, k)\right)^{2} \\
& =\frac{2 n+1}{4 \pi} \int_{\Omega} \int_{\Omega}(\widetilde{\Lambda F})(\xi)(\widetilde{\Lambda F})(\eta) P_{n}(\xi \cdot \eta) d \omega(\xi) d \omega(\eta),
\end{aligned}
$$


$n=0,1, \ldots$. According to Parseval's identity we clearly have

$$
\|\widetilde{\Lambda F}\|_{\mathcal{L}^{2}(\Omega)}^{2}=\sum_{n=0}^{\infty} \operatorname{var}_{n}(\widetilde{\Lambda F}) .
$$

Physical devices do not transmit spherical harmonics of arbitrarily high frequency without severe attenuation. The 'transfer' $\Lambda^{\wedge}(n)$ usually tends to zero with increasing $n$. It follows that the amplitude spectra of the responses (observations) to functions (signals) of finite $\mathcal{L}^{2}(\Omega)$-energy are negligibly small beyond some finite frequency. Thus, both because of the frequency limiting nature of the used devices and because of the nature of the 'transmitted signals', the geoscientist is soon led to consider bandlimited functions. These are the functions $\tilde{G} \in \mathcal{L}^{2}(\Omega)$, whose 'amplitude spectra' vanish for all $n>N\left(N \in \mathbb{N}_{0}\right.$, fixed). In other words, $\operatorname{var}_{n}(\tilde{G})=0, \quad n>N$. A function $\tilde{G}$ with $\tilde{G}^{\wedge}(n, k)=0$ for $n>N, k=1, \ldots, 2 n+1$, is said to be bandlimited with the band $N$. Furthermore, $\tilde{G} \in \mathcal{L}^{2}(\Omega)$ is said to be locally supported (spacelimited) with spacewidth $\rho$ around an axis $\eta \in \Omega$, if the function $\tilde{G}$ vanishes on the set of all $\xi \in \Omega$ with $-1 \leq \xi \cdot \eta \leq \rho$.

Bandlimited functions are of polynomial nature and, therefore, infinitely often differentiable everywhere on $\Omega$. Moreover, it is clear that $\tilde{G}$ is an analytic function. From the analyticity it follows immediately that a non-trivial bandlimited function cannot vanish on any (non-degenerate) subset of $\Omega$. The only function that is both bandlimited and spacelimited is the trivial function. Numerical analysis would like to deal with spacelimited functions. But as we have seen, such a function (signal) of finite (space) support cannot be bandlimited, it must allow signal degree variances of arbitrarily large degrees $n$. Thus there is a dilemma of seeking functions that are somehow concentrated in both space and frequency (i.e. angular momentum). The uncertainty principle (see W. Freeden (1999), W. Freeden, V. MicheL (1999)) is a way of mathematically expressing the impossibility of simultaneous confinement of a function (signal) to space and frequency.

\subsection{Degree Error Covariances}

The error theory is based on the spectral degree and order error covariance

$$
\operatorname{cov}_{n, k}(K)=\int_{\Omega} \int_{\Omega} K(\xi \cdot \eta) Y_{n, k}(\xi) Y_{n, k}(\eta) d \omega(\xi) d \omega(\eta)
$$


and the spectral degree error covariance

$$
\operatorname{cov}_{n}(K)=\sum_{k=1}^{2 n+1} \int_{\Omega} \int_{\Omega} K(\xi \cdot \eta) Y_{n, k}(\xi) Y_{n, k}(\eta) d \omega(\xi) d \omega(\eta)
$$

The Funk-Hecke formula yields

$$
\operatorname{cov}_{n, k}(K)=K^{\wedge}(n) .
$$

In other words, the spectral degree and order error covariance is simply the Legendre coefficient of the kernel $K$. Moreover, from the Funk-Hecke formula we are able to deduce in connection with the addition theorem that for all $\xi \in \Omega$

$$
\begin{aligned}
\operatorname{cov}_{n}(K) & =\frac{2 n+1}{4 \pi} \int_{\Omega} \int_{\Omega} K(\xi \cdot \eta) P_{n}(\xi \cdot \eta) d \omega(\xi) d \omega(\eta) \\
& =(2 n+1) K^{\wedge}(n) .
\end{aligned}
$$

In conclusion,

$$
\operatorname{cov}_{n, k}(K)=\frac{\operatorname{cov}_{n}(K)}{2 n+1}=K^{\wedge}(n)
$$

for $n=0,1, \ldots$

\subsection{Examples of Spectral Error Covariances}

To make the preceding considerations more concrete we would like to list two particularly important examples:

(1) Bandlimited white noise. Suppose that for some $n_{K} \in \mathbb{N}_{0}$

$$
K^{\wedge}(n)=\left\{\begin{array}{ccc}
\frac{\sigma^{2}}{\left(n_{K}+1\right)^{2}} & , \quad n \leq n_{K} \\
0 & , n>n_{K} .
\end{array}\right.
$$

The kernel reads as follows:

$$
\begin{aligned}
K(\xi \cdot \eta) & =\sum_{n=0}^{\infty} \frac{2 n+1}{4 \pi} K^{\wedge}(n) P_{n}(\xi \cdot \eta) \\
& =\frac{\sigma^{2}}{\left(n_{K}+1\right)^{2}} \sum_{n=0}^{n_{K}} \frac{2 n+1}{4 \pi} P_{n}(\xi \cdot \eta) .
\end{aligned}
$$


Note that this sum is a truncated Dirac functional. It is known (see e.g. N.N. LEBEDEW (1973)) that for $(\xi, \eta) \in \Omega \times \Omega$

$$
((\xi \cdot \eta)-1) K(\xi \cdot \eta)=\frac{\sigma^{2}}{4 \pi\left(n_{K}+1\right)}\left(P_{n_{K}+1}(\xi \cdot \eta)-P_{n_{K}}(\xi \cdot \eta)\right) .
$$

(2) Non-bandlimited colored noise. Assume that $K: \Omega \times \Omega \rightarrow \mathbb{R}$ is given in such a way that $K^{\wedge}(n)>0$ for an infinite number of non-negative integers $n$, the integral $\int_{-1}^{\delta} K(t) d t$ is sufficiently small (for some $\delta \in(1-\varepsilon, 1)$ for some $\varepsilon>0)$, and $K(\xi \cdot \xi)$ coincides with $\sigma^{2}$ for all $\xi \in \Omega$.

Geophysically relevant examples are the following kernels:

(i) $K(\xi \cdot \eta)=\frac{\sigma^{2}}{\exp (-c)} \exp (-c(\xi \cdot \eta)), \quad(\xi, \eta) \in \Omega \times \Omega$,

where $c$ is to be understood as the inverse spherical correlation length (first degree Gauß-Markov model).

(ii) $K(\xi \cdot \eta)=\frac{\sigma^{2}}{\left(L_{\rho_{J^{*}}}^{(k)}\right)^{(2)}(1)}\left(L_{\rho_{J^{*}}}^{(k)}\right)^{(2)}(\xi \cdot \eta), \quad(\xi, \eta) \in \Omega \times \Omega$,

for some sufficiently large $J^{*} \in \mathbb{N}$ (model of small correlation length).

The Legendre coefficients of the aforementioned kernels are calculable by recursion (as shown in W. FREEDEN et al. (1998)).

\subsection{Spectral Estimation}

In Section 2.2 we introduced the signal degree variances, whereas Section 2.3 was concerned with the introduction of the spectral error covariances. Now we are in position to compare the signal spectrum with that of the noise.

Signal and noise spectrum 'intersect' at the so-called degree resolution set $\mathcal{N}_{\text {res }}$ (with $\mathcal{N}_{\text {res }} \subset \mathcal{N}$ ). We distinguish the following cases:

(i) signal dominates noise

$$
\operatorname{var}_{n, k}(\widetilde{\Lambda F}) \geq \operatorname{cov}_{n, k}(K), \quad(n, k) \in \mathcal{N}_{\text {res }},
$$

(ii) noise dominates signal

$$
\operatorname{var}_{n, k}(\widetilde{\Lambda F})<\operatorname{cov}_{n, k}(K), \quad(n, k) \notin \mathcal{N}_{\text {res }} .
$$

Filtering is achieved by convolving a kernel $H \in \mathcal{L}^{2}[-1,+1]$ with the 'symbol' $\left\{H^{\wedge}(n)\right\}_{n \in \mathbb{N}_{0}}$ against $\widetilde{\Lambda F}$ :

$$
\widehat{\Lambda F}=\int_{\Omega} H(\cdot \eta) \widetilde{\Lambda F}(\eta) d \omega(\eta)
$$


(' $\wedge$ ' denotes 'estimated'). In spectral language this reads

$$
\widehat{\Lambda F}(n, k)=H^{\wedge}(n) \widetilde{\Lambda F}(n, k)
$$

for $n=0,1, \ldots ; k=1, \ldots, 2 n+1$.

Two important types of filtering are as follows:

(i) Spectral thresholding

$$
\widehat{\Lambda F}=\sum_{n=0}^{\infty} \sum_{k=1}^{2 n+1} I_{\mathcal{N}_{\text {res }}}(n, k) H^{\wedge}(n)(\widetilde{\Lambda F})^{\wedge}(n, k) Y_{n, k},
$$

where $I_{A}$ denotes the indicator function of the set $A$. This approach represents a 'keep or kill' filtering, where the signal dominated coefficients are filtered by $H \in \mathcal{L}^{2}[-1,+1]$, and the noise dominated coefficients are set to zero. This thresholding can be thought of as a non-linear operator on the set of coefficients, resulting in a set of estimated coefficients.

As a special filter we mention the ideal low-pass (Shannon) filter $H$ of the form

$$
H^{\wedge}(n)= \begin{cases}1, & n \leq n_{\mathrm{res}} \\ 0, & n>n_{\mathrm{res}}\end{cases}
$$

where $n_{\text {res }}=\inf _{n}\left\{(n, k) \in \mathcal{N}_{\text {res }}\right\}$. In that case all frequencies $n \leq n_{\text {res }}$ are allowed to pass, whereas all frequencies $n>n_{\text {res }}$ are completely eliminated.

(ii) Wiener-Kolmogorov filtering. Now we choose

$$
\widehat{\Lambda F}=\sum_{n=0}^{\infty} \sum_{k=1}^{2 n+1} H^{\wedge}(n)(\widetilde{\Lambda F})^{\wedge}(n, k) Y_{n, k}
$$

with

$$
H^{\wedge}(n)=\frac{\operatorname{var}_{n}(\widetilde{\Lambda F})}{\operatorname{var}_{n}(\widetilde{\Lambda F})+\operatorname{cov}_{n}(K)}, \quad n \in \mathbb{N}_{0} .
$$

This filter produces an optimal weighting between signal and noise (provided that complete independence of signal and noise is assumed). Note the similarity to the Tikhonov Singular Integral in (51).

For more details about filtering in physical geodesy the reader is referred to E.W. Grafarend (1982), H. Moritz (1980), R. Rummel (1997), F. SAnsò, R. Rummel (1997). 


\section{Multiscale Signal-to-Noise Response}

Consider a sequence $\left\{\Phi_{\rho_{j}}\right\}_{j \in \mathbb{Z}}$ constituting an approximate identity in $\mathcal{L}^{2}(\Omega)$ (as introduced in Section 1.3). Then an 'output signal' $\tilde{G} \in \mathcal{L}^{2}(\Omega)$ of an operator $\Lambda$ can be represented in multiscale approximation as follows

$$
\tilde{G}=\sum_{j=-\infty}^{+\infty} \int_{\Omega} \Psi_{\rho_{j}}^{(2)}(\cdot \eta) \tilde{G}(\eta) d \omega(\eta)
$$

where the equality is understood in $\|\cdot\|_{\mathcal{L}^{2}(\Omega)}$-sense. This result is equivalent to the identity

$$
\lim _{N \rightarrow \infty}\left\|\widetilde{\Lambda F}-\left((\widetilde{\Lambda F})_{J_{0}}+\sum_{j=J_{0}}^{N} \int_{\Omega} \Psi_{\rho_{j}}^{(2)}(\cdot \eta)(\widetilde{\Lambda F})(\eta) d \omega(\eta)\right)\right\|_{\mathcal{L}^{2}(\Omega)}=0
$$

for every $J_{0} \in \mathbb{Z}$. In terms of spherical harmonics we easily obtain

$$
\int_{\Omega} \Psi_{\rho_{j}}^{(2)}(\cdot \eta)(\widetilde{\Lambda F})(\eta) d \omega(\eta)=\sum_{n=0}^{\infty} \sum_{k=1}^{2 n+1}\left((\widetilde{\Lambda F})^{\wedge}(n, k)\right)\left(\Psi_{\rho_{j}}^{\wedge}(n)\right)^{2} Y_{n, k},
$$

where we have set

$$
\Psi_{\rho_{j}}^{(2)}(\xi \cdot \eta)=\Phi_{\rho_{j+1}}^{(2)}(\xi \cdot \eta)-\Phi_{\rho_{j}}^{(2)}(\xi \cdot \eta),
$$

$j \in \mathbb{Z},(\xi, \eta) \in \Omega \times \Omega$. Note that we restrict ourselves to the so-called Pwavelet concept (for other wavelet approaches see FREEDEN et al. (1998)).

\subsection{Scale and Position Variances}

Denote by $\mathcal{L}^{2}(\mathbb{Z} \times \Omega)$ the space of functions $H: \mathbb{Z} \times \Omega \rightarrow \mathbb{R}$ satisfying

$$
\sum_{j=-\infty}^{\infty} \int_{\Omega}(H(j ; \eta))^{2} d \omega(\eta)<\infty
$$

$\mathcal{L}^{2}(\mathbb{Z} \times \Omega)$ is a Hilbert space equipped with the inner product

$$
\left(H_{1}, H_{2}\right)_{\mathcal{L}^{2}(\mathbb{Z} \times \Omega)}=\sum_{j=-\infty}^{+\infty} \int_{\Omega} H_{1}(j ; \eta) H_{2}(j ; \eta) d \omega(\eta)
$$


corresponding to the norm

$$
\|H\|_{\mathcal{L}^{2}(\mathbb{Z} \times \Omega)}=\left(\sum_{j=-\infty}^{+\infty} \int_{\Omega}(H(j ; \eta))^{2} d \omega(\eta)\right)^{1 / 2} .
$$

Consider a kernel $\left\{\Phi_{\rho_{j}}\right\}_{j \in \mathbb{Z}}$ constituting an approximate identity in $\mathcal{L}^{2}(\Omega)$. From the multiscale formulation of an 'output function' $\tilde{G}=\widetilde{\Lambda F} \in \mathcal{L}^{2}(\Omega)$ (see Section 1.3) we immediately obtain

$$
\begin{aligned}
& (\widetilde{\Lambda F}, \widetilde{\Lambda F})_{\mathcal{L}^{2}(\Omega)} \\
& =\sum_{n=0}^{\infty} \sum_{k=1}^{2 n+1}\left((\widetilde{\Lambda F})^{\wedge}(n, k)\right)^{2} \sum_{j=-\infty}^{+\infty}\left(\left(\Psi_{\rho_{j}}\right)^{\wedge}(n)\right)^{2} \\
& =\sum_{j=-\infty}^{+\infty} \int_{\Omega}\left(\int_{\Omega}(\widetilde{\Lambda F})(\xi) \Psi_{\rho_{j}}(\eta \cdot \xi) d \omega(\xi)\right)^{2} d \omega(\eta) \\
& =\sum_{j=-\infty}^{+\infty} \int_{\Omega}\left(\int_{\Omega} \int_{\Omega}(\widetilde{\Lambda F})(\xi)(\widetilde{\Lambda F})(\zeta) \Psi_{\rho_{j}}(\xi \cdot \eta) \Psi_{\rho_{j}}(\zeta \cdot \eta) d \omega(\xi) d \omega(\zeta)\right) d \omega(\eta)
\end{aligned}
$$

The signal scale and space variance of $\widetilde{\Lambda F}$ at position $\eta \in \Omega$ and scale $j \in \mathbb{Z}$ is defined by

$$
\operatorname{var}_{j ; \eta}^{2}(\widetilde{\Lambda F})=\int_{\Omega} \int_{\Omega}(\widetilde{\Lambda F})(\xi)(\widetilde{\Lambda F})(\zeta) \Psi_{\rho_{j}}(\xi \cdot \eta) \Psi_{\rho_{j}}(\zeta \cdot \eta) d \omega(\xi) d \omega(\zeta) .
$$

The signal scale variance of $\widetilde{\Lambda F}$ is defined by

$$
\operatorname{var}_{j}^{2}(\widetilde{\Lambda F})=\int_{\Omega} \operatorname{var}_{j ; \eta}^{2}(\widetilde{\Lambda F}) d \omega(\eta)
$$

Obviously, we have

$$
\begin{aligned}
\|\widetilde{\Lambda F}\|_{\mathcal{L}^{2}(\Omega)}^{2} & =\sum_{j=-\infty}^{+\infty} \operatorname{var}_{j}^{2}(\widetilde{\Lambda F}) \\
& =\sum_{j=-\infty}^{+\infty} \int_{\Omega} \operatorname{var}_{j ; \eta}^{2}(\widetilde{\Lambda F}) d \omega(\eta) \\
& =\left\|\operatorname{var}_{\cdot, \cdot}(\widetilde{\Lambda F})\right\|_{\mathcal{L}^{2}(\mathbb{Z} \times \Omega)}^{2} .
\end{aligned}
$$


Expressed in the spectral language of spherical harmonics we get

$$
\begin{aligned}
& \operatorname{var}_{j ; \cdot}^{2}(\widetilde{\Lambda F})= \\
& =\sum_{n=0}^{\infty} \sum_{k=1}^{2 n+1} \sum_{m=0}^{\infty} \sum_{l=1}^{2 m+1} \Psi_{\rho_{j}}^{\wedge}(n) \Psi_{\rho_{j}}^{\wedge}(m)(\widetilde{\Lambda F})^{\wedge}(n, k) . \\
& \cdot(\widetilde{\Lambda F})^{\wedge}(m, l) Y_{n, k}(\cdot) Y_{m, l}(\cdot) .
\end{aligned}
$$

Consequently,

$$
\operatorname{var}_{j}^{2}(\widetilde{\Lambda F})=\int_{\Omega} \operatorname{var}_{j ; \eta}^{2}(\widetilde{\Lambda F}) d \omega(\eta)=\sum_{n=0}^{\infty} \sum_{k=1}^{2 n+1}\left(\Psi_{\rho_{j}}^{\wedge}(n)\right)^{2}\left((\widetilde{\Lambda F})^{\wedge}(n, k)\right)^{2}
$$

With the convention $\mathcal{Z}=\mathbb{Z} \times \Omega$ we formally write

$$
\|\widetilde{\Lambda F}\|_{\mathcal{L}^{2}(\Omega)}^{2}=\sum_{(j ; \eta) \in \mathcal{Z}} \operatorname{var}_{j ; \eta}^{2}(\widetilde{\Lambda F}) d \omega(\eta)=\left\|\operatorname{var}_{., .}(\widetilde{\Lambda F})\right\|_{\mathcal{L}^{2}(\mathcal{Z})} .
$$

We mention that the Beppo-Levi Theorem justifies the notation $\mathbb{E}$ in (96), as we are allowed to interchange integration and summation. Note that all integrations are understood in the Lebesgue-sense.

\subsection{Noise Model}

Let $K:(\xi, \eta) \mapsto K(\xi \cdot \eta),(\xi, \eta) \in \Omega \times \Omega$, satisfy the conditions $(\mathrm{C} 1)$ and (C2) stated in Section 2.1. The error theory is based on the scale and space error covariance at $\eta \in \Omega$

$$
\operatorname{cov}_{j ; \eta}(K)=\int_{\Omega} \int_{\Omega} K(\xi \cdot \zeta) \Psi_{\rho_{j}}^{(2)}(\xi \cdot \eta) \Psi_{\rho_{j}}^{(2)}(\zeta \cdot \eta) d \omega(\xi) d \omega(\zeta), \quad \eta \in \Omega
$$

The scale error covariance is defined by

$$
\operatorname{cov}_{j}(K)=\int_{\Omega} \operatorname{cov}_{j ; \eta}(K) d \omega(\eta)
$$

Note that

$$
\operatorname{cov}_{j}(K)=4 \pi \operatorname{cov}_{j ; \eta}(K)
$$


for all $\eta \in \Omega$. We obviously have

$$
\operatorname{cov}_{j ; \eta}(K)=\sum_{n=0}^{\infty} \frac{2 n+1}{4 \pi} K^{\wedge}(n)\left(\Psi_{\rho_{j}}^{\wedge}(n)\right)^{4} .
$$

It is clear from our stochastic model that the scale error covariance cannot be dependent on the position $\eta \in \Omega$. This is also indicated by the spectral formula:

$$
\operatorname{cov}_{j ; \eta}(K)=\frac{1}{4 \pi} \sum_{n=0}^{\infty} \operatorname{cov}_{n}(K)\left(\Psi_{\rho_{j}}^{\wedge}(n)\right)^{4} .
$$

\subsection{Scale and Space Estimation}

Signal and noise scale 'intersect' at the so-called scale and space resolution set $\mathcal{Z}_{\text {res }} \subset \mathcal{Z}$. We distinguish the following cases:

(i) signal dominates noise

$$
\operatorname{var}_{j ; \eta}(\widetilde{\Lambda F}) \geq \operatorname{cov}_{j ; \eta}(K), \quad(j ; \eta) \in \mathcal{Z}_{\text {res }}
$$

(ii) noise dominates signal

$$
\operatorname{var}_{j ; \eta}(\widetilde{\Lambda F})<\operatorname{cov}_{j ; \eta}(K), \quad(j ; \eta) \notin \mathcal{Z}_{\text {res }} .
$$

Via the multiscale reconstruction formula the (filtered) $J$-level approximation of the error-affected function $\widetilde{\Lambda F}$ reads as follows

$$
(\widetilde{\Lambda F})_{J}=\sum_{j=-\infty}^{J} \int_{\Omega} \Psi_{\rho_{j}}^{(2)}(\cdot \eta)(\widetilde{\Lambda F})(\eta) d \omega(\eta)
$$

In shorthand notation,

$$
(\widetilde{\Lambda F})_{J}=\sum_{\substack{(j ; \eta) \in \mathcal{Z} \\ j \leq J}} \Psi_{\rho_{j}}^{(2)}(\cdot \eta)(\widetilde{\Lambda F})(\eta) d \omega(\eta)
$$

For $J$ sufficiently large, $\widetilde{\Lambda F}$ is well-represented by $(\widetilde{\Lambda F})_{J}$. In other words, all the higher-level coefficients are regarded as being negligible, i.e. $(\widetilde{\Lambda F})_{J} \simeq$ $\widetilde{\Lambda F}$. 


\section{Selective Multiscale Reconstruction}

Similar to what is known in taking Fourier approximation, we are able to take multiscale approximation by replacing the (unknown) error-free function $\Lambda F$ of the representation

$$
\begin{aligned}
(\Lambda F)_{J} & =\int_{\Omega} \Phi_{\rho_{J_{0}}}^{(2)}(\cdot \zeta)(\Lambda F)(\zeta) d \omega(\zeta) \\
& +\sum_{j=J_{0}}^{J-1} \int_{\Omega} \Psi_{\rho_{j}}^{(2)}(\cdot \zeta)(\Lambda F)(\zeta) d \omega(\zeta)
\end{aligned}
$$

by (an estimate from) the error-affected function $\widetilde{\Lambda F}$ such as

$$
\begin{aligned}
(\widetilde{\Lambda F})_{J} & =\int_{\Omega} \Phi_{\rho_{J_{0}}}^{(2)}(\cdot \zeta)(\widetilde{\Lambda F})(\zeta) d \omega(\zeta) \\
& +\sum_{j=J_{0}}^{J-1} \int_{\Omega} \Psi_{\rho_{j}}^{(2)}(\cdot \zeta)(\widetilde{\Lambda F})(\zeta) d \omega(\zeta)
\end{aligned}
$$

$J>J_{0}$. Computing the following coefficients at position $\eta \in \Omega$

$$
\begin{aligned}
v_{J_{0} ; \eta} & =\int_{\Omega} \Phi_{\rho_{J_{0}}}^{(2)}(\eta \cdot \zeta)(\Lambda F)(\zeta) d \omega(\zeta) \\
w_{j ; \eta} & =\int_{\Omega} \Psi_{\rho_{j}}^{(2)}(\eta \cdot \zeta)(\Lambda F)(\zeta) d \omega(\zeta), \quad j=J_{0}, \ldots, J-1,
\end{aligned}
$$

and

$$
\begin{aligned}
\tilde{v}_{J_{0} ; \eta} & =\int_{\Omega} \Phi_{\rho_{J_{0}}}^{(2)}(\eta \cdot \zeta)(\widetilde{\Lambda F})(\zeta) d \omega(\zeta) \\
\tilde{w}_{j ; \eta} & =\int_{\Omega} \Psi_{\rho_{j}}^{(2)}(\eta \cdot \zeta)(\widetilde{\Lambda F})(\zeta) d \omega(\zeta), \quad j=J_{0}, \ldots, J-1
\end{aligned}
$$

will, of course, require adequate methods of numerical integration on the sphere.

\subsection{Equidistributions}

Many integration techniques are known from the literature (for a survey on approximate integration on the sphere see, for example, W. FrEEDEN et al. (1998)). In what follows we base approximate integration on the concept of 
equidistribution. This concept is indeed of particular significance in future satellite geodesy and geomagnetics, where millions of data of sufficiently dense nodal widths on (nearly) circular, polar orbits will be provided by satellite missions (such as CHAMP, GRACE, GOCE).

In order to formulate suitable discretizations of the integrals $\tilde{v}_{J_{0} ; \eta}, \tilde{w}_{j ; \eta}$ we make the following restrictions on the nodal systems to be used.

A point system $X_{N}=\left\{\eta_{1}^{N}, \ldots, \eta_{N}^{N}\right\} \subset \Omega$ of $N$ points $\eta_{1}^{N}, \ldots, \eta_{N}^{N}$ is said to be an $\alpha$-equidistribution, if there is a partition of $\Omega$ into $N$ mutually disjoint measurable parts $\pi_{\eta_{1}^{N}}, \ldots, \pi_{\eta_{N}^{N}}$ of equal area $\frac{4 \pi}{N}$ with the property that each point $\eta_{i}^{N}, i \in\{1, \ldots, N\}$, is associated to $\pi_{\eta_{i}^{N}}$ in such a way that

$$
\sup _{\xi \in \pi_{\eta_{i}^{N}}^{N}}\left|\xi-\eta_{i}^{N}\right| \leq N^{-\alpha}
$$

where $\alpha$ is a positive constant.

Our approach now will be based on the additional, but not very restrictive assumption that all functions $\Phi_{\rho_{j}}^{(2)}(\cdot \eta) \widetilde{\Lambda F}, j=J_{0}, \ldots, J$, are Lipschitzcontinuous on $\Omega$. Then it follows that for every $\eta \in \Omega$

$$
\begin{aligned}
\tilde{w}_{j, \eta} & =\sum_{i=1}^{N_{j}} \int_{\eta_{i}^{N_{j}}} \Psi_{\rho_{j}}^{(2)}(\eta \cdot \zeta)(\widetilde{\Lambda F})(\zeta) d \omega(\zeta) \\
& =\frac{4 \pi}{N_{j}} \sum_{i=1}^{N_{j}} \Psi_{\rho_{j}}^{(2)}\left(\eta \cdot \eta_{i}^{N_{j}}\right)(\widetilde{\Lambda F})\left(\eta_{i}^{N_{j}}\right) \\
& +\sum_{i=1}^{N_{j}} \int_{\eta_{i}^{N_{j}}}\left(\Psi_{\rho_{j}}^{(2)}(\eta \cdot \zeta)(\widetilde{\Lambda F})(\zeta)-\Psi_{\rho_{j}}^{(2)}\left(\eta \cdot \eta_{i}^{N_{j}}\right)(\widetilde{\Lambda F})\left(\eta_{i}^{N_{j}}\right)\right) d \omega(\zeta)
\end{aligned}
$$

Consequently, for $j=J_{0}, \ldots, J-1$ and $\eta \in \Omega$, it follows from the Lipschitz continuity that

$$
\tilde{w}_{j ; \eta}=\frac{4 \pi}{N_{j}} \sum_{i=1}^{N_{j}} \Psi_{\rho_{j}}^{(2)}\left(\eta \cdot \eta_{i}^{N_{j}}\right)(\widetilde{\Lambda F})\left(\eta_{i}^{N_{j}}\right)+O\left(N_{j}^{-\alpha_{j}}\right),
$$

provided that $\left\{\eta_{1}^{N_{j}}, \ldots, \eta_{N_{j}}^{N_{j}}\right\} \subset \Omega$ is an $\alpha_{j}$-equidistribution. An analogous argument applies to $\tilde{v}_{J_{0} ; \eta}$. 
The investigations which follow will essentially be based on the observation that the values $\tilde{v}_{J_{0} ; \eta}, \tilde{w}_{j ; \eta}, j=J_{0}, \ldots, J-1$, can be determined by the following fomulae:

$$
\begin{aligned}
\tilde{v}_{J_{0} ; \eta} & \simeq \frac{4 \pi}{N_{J_{0}}} \sum_{i=1}^{N_{J_{0}}} \Phi_{\rho_{J_{0}}}^{(2)}\left(\eta \cdot \eta_{i}^{N_{J_{0}}}\right)(\widetilde{\Lambda F})\left(\eta_{i}^{N_{J_{0}}}\right), \\
\tilde{w}_{j ; \eta} & \simeq \frac{4 \pi}{N_{j}} \sum_{i=1}^{N_{j}} \Psi_{\rho_{j}}^{(2)}\left(\eta \cdot \eta_{i}^{N_{j}}\right)(\widetilde{\Lambda F})\left(\eta_{i}^{N_{j}}\right), \quad j=J_{0}, \ldots, J-1
\end{aligned}
$$

(' $\simeq$ ' always means that the error is assumed to be negligible for sufficiently large $N_{j}$ ).

\subsection{A Pyramid Scheme}

In accordance to the ideas developed by W. FREEDEN (1999) we now describe a pyramid scheme for the recursive computation of the integrals $\tilde{v}_{J_{0} ; \eta}$, $\tilde{w}_{j ; \eta}$ for $j=J_{0}, \ldots, J-1$.

Let $\left\{X_{N_{j}}\right\}, j=J_{0}, \ldots, J$, be a sequence of $\alpha_{j}$-equidistributions $X_{N_{j}}=$ $\left\{\eta_{1}^{N_{j}}, \ldots, \eta_{N_{j}}^{N_{j}}\right\}$ such that

$$
\begin{aligned}
& \Phi_{\rho_{j}}^{(2)}(\cdot \eta) * \widetilde{\Lambda F} \simeq \sum_{i=1}^{N_{j}} \Phi_{\rho_{j}}^{(2)}\left(\eta \cdot \eta_{i}^{N_{j}}\right) \tilde{a}_{i}^{N_{j}}, \quad j=J_{0}, \ldots, J, \\
& \Psi_{\rho_{j}}^{(2)}(\cdot \eta) * \widetilde{\Lambda F} \simeq \sum_{i=1}^{N_{j}} \Psi_{\rho_{j}}^{(2)}\left(\eta \cdot \eta_{i}^{N_{j}}\right) \tilde{a}_{i}^{N_{j}}, \quad j=J_{0}, \ldots, J-1,
\end{aligned}
$$

where the coefficients $\tilde{a}_{i}^{N_{j}}$ are given by

$$
\tilde{a}_{i}^{N_{j}}=\frac{4 \pi}{N_{j}} \widetilde{\Lambda F}\left(\eta_{i}^{N_{j}}\right)
$$

for $j=J_{0}, \ldots, J$.

What we are going to realize is a tree algorithm (pyramid scheme) with the following ingredients: Starting from a sufficiently large $J$ such that

$$
\widetilde{\Lambda F}(\eta) \simeq \Phi_{\rho_{J}}^{(2)}(\cdot \eta) * \widetilde{\Lambda F} \simeq \sum_{i=1}^{N_{J}} \Phi_{\rho_{J}}^{(2)}\left(\eta \cdot \eta_{i}^{N_{J}}\right) \tilde{a}_{i}^{N_{J}}, \quad \eta \in \Omega,
$$


we want to show that the coefficient vectors $\tilde{a}^{N_{j}}=\left(\tilde{a}_{1}^{N_{j}}, \ldots, \tilde{a}_{N_{j}}^{N_{j}}\right) \in \mathbb{R}^{N_{j}}$ $j=J_{0}, \ldots, J-1$, (being, of course, dependent on the function $\widehat{\Lambda F}$ under consideration) can be calculated such that the following statements hold true:

(i) The vectors $\tilde{a}^{N_{j}}, j=J_{0}, \ldots, J-1$, are obtainable by recursion, i.e.

$$
\tilde{a}_{i}^{N_{j-1}} \simeq \frac{4 \pi}{N_{j-1}} \sum_{l=1}^{N_{j}} \Phi_{\rho_{J}}^{(2)}\left(\eta_{i}^{N_{j-1}} \cdot \eta_{l}^{N_{j}}\right) \tilde{a}_{l}^{N_{j}} .
$$

(ii) For $j=J_{0}, \ldots, J$

$$
\Phi_{\rho_{j}}^{(2)}(\cdot \eta) * \widetilde{\Lambda F}=\sum_{i=1}^{N_{j}} \Phi_{\rho_{j}}^{(2)}\left(\eta \cdot \eta_{i}^{N_{j}}\right) \tilde{a}_{i}^{N_{j}},
$$

and for $j=J_{0}, \ldots, J-1$

$$
\Psi_{\rho_{j}}^{(2)}(\cdot \eta) * \widetilde{\Lambda F}=\sum_{i=1}^{N_{j}} \Psi_{\rho_{j}}^{(2)}\left(\eta \cdot \eta_{i}^{N_{j}}\right) \tilde{a}_{i}^{N_{j}} .
$$

Our considerations are divided into two parts, viz. the initial step concerning the scale level $J$ and the pyramid step establishing the recursion relation:

The Initial Step. For suitably large $J, \Phi_{\rho_{J}}^{(2)}(\eta) * \widetilde{\Lambda F}$ is sufficiently close to $(\widetilde{\Lambda F})(\eta)$ for all $\eta \in \Omega$. Formally spoken, the kernel $\Phi_{\rho_{J}}^{(2)}$ replaces the Dirac-functional $\delta$ as follows:

$$
\Phi_{\rho_{J}}^{(2)}(\cdot \eta) * \widetilde{\Lambda F} \simeq \widetilde{\Lambda F}(\eta)=\delta(\cdot \eta) * \widetilde{\Lambda F}
$$

This is the reason why the coefficients $\tilde{a}^{N_{J}}=\left(\tilde{a}_{1}^{N_{J}}, \ldots, \tilde{a}_{N_{J}}^{N_{J}}\right) \in \mathbb{R}^{N_{J}}$ are assumed to be given in the form

$$
\tilde{a}_{i}^{N_{J}}=\frac{4 \pi}{N_{J}}(\widetilde{\Lambda F})\left(\eta_{i}^{N_{J}}\right)
$$

Moreover, it is obvious that

$$
\Psi_{\rho_{J}}^{(2)}(\cdot \eta) * \widetilde{\Lambda F}=\sum_{i=1}^{N_{J}} \Psi_{\rho_{J}}^{(2)}\left(\eta \cdot \eta_{i}^{N_{J}}\right) \tilde{a}_{i}^{N_{J}}
$$


The Pyramid Step. An essential tool for the pyramid scheme is the fact that

$$
\tilde{a}_{i}^{N_{j}}=\frac{4 \pi}{N_{j}}(\widetilde{\Lambda F})\left(\eta_{i}^{N_{j}}\right) \simeq \frac{4 \pi}{N_{j}} \Phi_{\rho_{J}}^{(2)}\left(\cdot \eta_{i}^{N_{j}}\right) * \widetilde{\Lambda F}
$$

for $j=J_{0}, \ldots, J$. Thus it follows that

$$
\Phi_{\rho_{j}}^{(2)}(\cdot \eta) * \widetilde{\Lambda F} \simeq \sum_{i=1}^{N_{j}} \Phi_{\rho_{j}}^{(2)}\left(\eta \cdot \eta_{i}^{N_{j}}\right) \tilde{a}_{i}^{N_{j}} .
$$

From (128) we are easily able to verify (cf. W. FREEDEN (1999)) that

$$
(\widetilde{\Lambda F})^{\wedge}(n, k)=\sum_{i=1}^{N_{j}} \tilde{a}_{i}^{N_{j}} Y_{n, k}\left(\eta_{i}^{N_{j}}\right)
$$

for $n=0,1, \ldots ; k=1, \ldots, 2 n+1$. Note that the coefficients $\tilde{a}_{i}^{N_{j}}$ are independent of the choice of the kernel $\left\{\Phi_{\rho_{j}}^{(2)}\right\}$. In particular, we have

$$
\Psi_{\rho_{j}}^{(2)}(\cdot \eta) * \widetilde{\Lambda F} \simeq \sum_{i=1}^{N_{j}} \Psi_{\rho_{j}}^{(2)}\left(\eta \cdot \eta_{i}^{N_{j}}\right) \tilde{a}_{i}^{N_{j}} .
$$

We now come to the recursion step. On the one hand side we have from (120)

$$
\Phi_{\rho_{J}}^{(2)}(\cdot \eta) * \widetilde{\Lambda F} \simeq \sum_{i=1}^{N_{j}} \Phi_{\rho_{J}}^{(2)}\left(\eta \cdot \eta_{i}^{N_{j}}\right) \tilde{a}_{i}^{N_{j}} .
$$

On the other hand side we have

$$
\Phi_{\rho_{j-1}}^{(2)}(\cdot \eta) * \widetilde{\Lambda F} \simeq \sum_{i=1}^{N_{j-1}} \Phi_{\rho_{j-1}}^{(2)}\left(\eta \cdot \eta_{i}^{N_{j-1}}\right) \tilde{a}_{i}^{N_{j-1}}
$$

with coefficients $\tilde{a}_{i}^{N_{j-1}}$ given by

$$
\tilde{a}_{i}^{N_{j-1}} \simeq \frac{4 \pi}{N_{j-1}} \Phi_{\rho_{J}}^{(2)}\left(\cdot \eta_{i}^{N_{j-1}}\right) * \widetilde{\Lambda F}
$$

Inserting (131) into (133) we find (121). In other words, the coefficients $\tilde{a}_{i}^{N_{j-1}}$ can be calculated recursively starting from the level $J$. Moreover, the 
coefficients are independent of the special choice of the kernel. This finally leads us to the formulae

$$
\Phi_{\rho_{j}}^{(2)}(\cdot \eta) * \widetilde{\Lambda F} \simeq \sum_{i=1}^{N_{j}} \Phi_{\rho_{j}}^{(2)}\left(\eta \cdot \eta_{i}^{N_{j}}\right) \tilde{a}_{i}^{N_{j}}, \quad j=J_{0}, \ldots, J,
$$

and

$$
\Psi_{\rho_{j}}^{(2)}(\cdot \eta) * \widetilde{\Lambda F} \simeq \sum_{i=1}^{N_{j}} \Psi_{\rho_{j}}^{(2)}\left(\eta \cdot \eta_{i}^{N_{j}}\right) \tilde{a}_{i}^{N_{j}}, \quad j=J_{0}, \ldots, J-1,
$$

with coefficients $\tilde{a}_{i}^{N_{j}}$ given by (125) and (121).

This recursion procedure leads us to the following decomposition scheme:

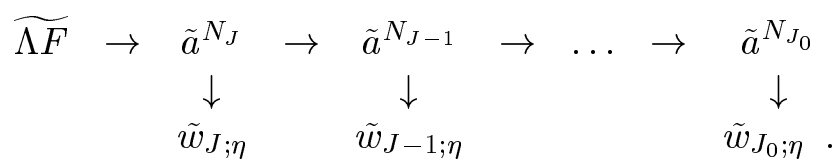

The coefficient vectors $\tilde{a}^{N_{J_{0}}}, \tilde{a}^{N_{J_{0}+1}}, \ldots$ allow the following reconstruction scheme of $\widetilde{\Lambda F}$ :

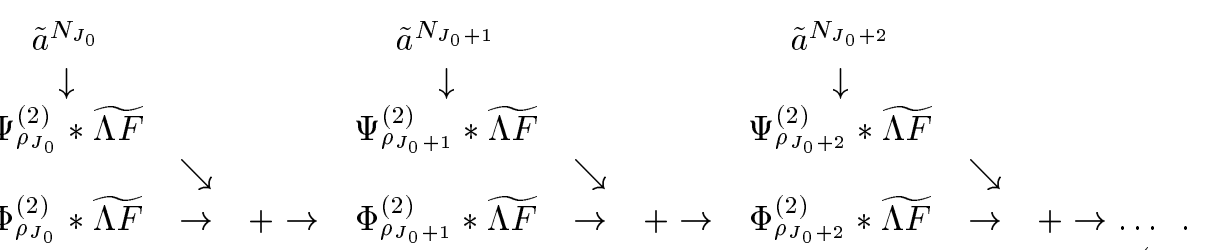

It is worth mentioning that the coefficient vectors $\tilde{a}^{N_{j}}$ do not depend on the special choice of the scaling function $\left\{\Phi_{\rho_{j}}^{(2)}\right\}_{j \in \mathbb{Z}}$ in $\mathcal{L}^{2}(\Omega)$. Moreover, the coefficients can be used to calculate the wavelet transforms $\Psi_{\rho_{j}}(\cdot \eta) * \widetilde{\Lambda F}$ for $j=J_{0}, \ldots, J-1$ and all $\eta \in \Omega$.

\subsection{Empirical Multiscale Coefficients}

In Section 2.1 we introduced the error model such that for any $\xi \in \Omega$ $\tilde{\epsilon}(\xi)$ is $N\left(0, \sigma^{2}\right)$-distributed, i.e. $E[(\widetilde{\Lambda F})(\xi)]=(\Lambda F)(\xi), \operatorname{var}[(\widetilde{\Lambda F})(\xi)]=$ $\operatorname{var}[\tilde{\epsilon}(\xi)]=\sigma^{2}$. Furthermore, we have $\operatorname{cov}[\tilde{\epsilon}(\xi), \tilde{\epsilon}(\eta)]=K(\xi \cdot \eta)$ for $\xi, \eta \in \Omega$. 
THEOREM 4.1.For $j=J_{0}, \ldots, J-1$, and all $\eta \in \Omega$, the expectation value and variance of $\tilde{w}_{j ; \eta}$ satisfy

$$
\begin{aligned}
E\left[\tilde{w}_{j ; \eta}\right] & =w_{j ; \eta}, \\
\operatorname{var}\left[\tilde{w}_{j ; \eta}\right] & =\operatorname{cov}_{j ; \eta}(K) .
\end{aligned}
$$

Proof. The Fubini Theorem of measure theory allows us to interchange integration and the calculation of the expectation values, as the considered Lebesgue integrals are finite. In doing so we obtain

$$
\begin{aligned}
E\left[\tilde{w}_{j ; \eta}\right] & =\int_{\Omega} \Psi_{\rho_{j}}^{(2)}(\eta \cdot \xi) E[(\widetilde{\Lambda F})(\xi)] d \omega(\xi) \\
& =w_{j ; \eta}
\end{aligned}
$$

Next we see that

$$
\begin{aligned}
\operatorname{var} & {\left[\tilde{w}_{j ; \eta}\right]=E\left[\left(\tilde{w}_{j ; \eta}-E\left[\tilde{w}_{j ; \eta}\right]\right)^{2}\right]=} \\
& =E\left[\int_{\Omega} \int_{\Omega} \Psi_{\rho_{j}}^{(2)}(\eta \cdot \xi) \Psi_{\rho_{j}}^{(2)}(\eta \cdot \zeta) \tilde{\epsilon}(\xi) \tilde{\epsilon}(\zeta) d \omega(\xi) d \omega(\zeta)\right] \\
& =\int_{\Omega} \int_{\Omega} \Psi_{\rho_{j}}^{(2)}(\eta \cdot \xi) \Psi_{\rho_{j}}^{(2)}(\eta \cdot \zeta) E[\tilde{\epsilon}(\xi) \tilde{\epsilon}(\zeta)] d \omega(\xi) d \omega(\zeta) \\
& =\int_{\Omega} \int_{\Omega} \Psi_{\rho_{j}}^{(2)}(\eta \cdot \xi) \Psi_{\rho_{j}}^{(2)}(\eta \cdot \zeta) K(\xi \cdot \zeta) d \omega(\xi) d \omega(\zeta) \\
& =\operatorname{cov}_{j ; \eta}(K) .
\end{aligned}
$$

This is the desired result.

Following the ideas proposed by D.L. Donoho, I.M. Johnstone (1994, 1995), each empirical coefficient consists of a certain amount of noise, but only relatively few consist of significant signal. Therefore, the canonical question is to ask which of the coefficients contain significant signal, and which are mostly noise. Once we have chosen the set of coefficients containing significant signal, some attempt might be made to remove the noise from each empirical coefficient. The idea of thresholding represents a very useful method to estimate $(\widetilde{\Lambda F})_{J}$. 


\subsection{Scale Thresholding}

Since the large 'true' coefficients are the ones that should be included in a selective reconstruction, in estimating an unknown function it is natural to include only coefficients larger than some specified threshold value.

In our context a 'larger' coefficient is taken to mean one that satisfies for $j=J_{0}, \ldots, J$ and $i=1, \ldots, N_{j}$

$$
\begin{aligned}
& \left(\tilde{a}_{i}^{N_{j}}\right)^{2} \\
& \simeq\left(\frac{4 \pi}{N_{j}}\right)^{2} \int_{\Omega} \int_{\Omega}(\widetilde{\Lambda F})(\xi)(\widetilde{\Lambda F})(\zeta) \Phi_{\rho_{j}}^{(2)}\left(\xi \cdot \eta_{i}^{N_{j}}\right) \Phi_{\rho_{j}}^{(2)}\left(\zeta \cdot \eta_{i}^{N_{j}}\right) d \omega(\xi) d \omega(\zeta) \\
& \geq\left(\frac{4 \pi}{N_{j}}\right)^{2} \int_{\Omega} \int_{\Omega} K(\xi \cdot \zeta) \Phi_{\rho_{j}}^{(2)}\left(\xi \cdot \eta_{i}^{N_{j}}\right) \Phi_{\rho_{j}}^{(2)}\left(\zeta \cdot \eta_{i}^{N_{j}}\right) d \omega(\xi) d \omega(\zeta) \\
& =k_{j}^{2}
\end{aligned}
$$

where we have used the abbreviation

$$
k_{j}=\frac{4 \pi}{N_{j}}\left(\sum_{n=0}^{\infty} \frac{2 n+1}{4 \pi} K^{\wedge}(n)\left(\Phi_{\rho_{j}}^{\wedge}(n)\right)^{4}\right)^{1 / 2} .
$$

Remark. In particular for bandlimited white noise of the form (cf. R. RUMMEL (1992))

$$
K(\eta \cdot \xi)=\frac{\sigma^{2}}{4 \pi} P_{0}(\eta \cdot \xi)=\frac{\sigma^{2}}{4 \pi},
$$

$(\eta, \xi) \in \Omega \times \Omega$, we find

$$
k_{j}=\frac{2 \sqrt{\pi}}{N_{j}} \sigma\left(\Phi_{\rho_{j}}^{\wedge}(0)\right)^{2}, \quad j=J_{0}, \ldots, J
$$

For the given threshold values $k_{j}$ such an estimator can be written in explicit form:

$$
\begin{aligned}
(\widehat{\Lambda F})_{J} & =\sum_{i=1}^{N_{J_{0}}} I_{\left\{\left|\tilde{a}_{i}^{N_{J_{0}}}\right| \geq k_{J_{0}}\right\}} \Phi_{\rho_{J_{0}}}^{(2)}\left(\cdot \eta_{i}^{N_{J_{0}}}\right) \tilde{a}_{i}^{N_{J_{0}}} \\
& +\sum_{j=J_{0}}^{J-1} \sum_{i=1}^{N_{j}} I_{\left\{\left|\tilde{a}_{i}{ }_{j}\right| \geq k_{j}\right\}} \Psi_{\rho_{j}}^{(2)}\left(\cdot \eta_{i}^{N_{j}}\right) \tilde{a}_{i}^{N_{j}} .
\end{aligned}
$$


In other words, the large coefficients (relative to the threshold $k_{j}, j=$ $\left.J_{0}, \ldots, J-1\right)$ are kept intact and the small coefficients are set to zero. Motivated by our results explained in Section 5.3 the thresholding will be performed on $\tilde{v}_{J_{0} ; \eta}$ and $\tilde{w}_{j ; \eta}, j=J_{0}, \ldots, J-1$. The thresholding estimators of the true coefficients $v_{J_{0} ; \eta}, w_{j ; \eta}$ can thus be written in the form

$$
\begin{array}{r}
\hat{v}_{J_{0} ; \eta}=\sum_{i=1}^{N_{J_{0}}} \delta_{k_{J_{0}}}\left(\tilde{a}_{i}^{N_{J_{0}}}\right) \Phi_{\rho_{J_{0}}}^{(2)}\left(\eta \cdot \eta_{i}^{N_{J_{0}}}\right), \\
\hat{w}_{j ; \eta}=\sum_{i=1}^{N_{j}} \delta_{k_{j}}\left(\tilde{a}_{i}^{N_{j}}\right) \Psi_{\rho_{j}}^{(2)}\left(\eta \cdot \eta_{i}^{N_{j}}\right),
\end{array}
$$

where the function $\delta_{\lambda}$ is the hard thresholding function

$$
\delta_{\lambda}^{\text {hard }}(x)= \begin{cases}x & \text { if }|x| \geq \lambda \\ 0 & \text { otherwise }\end{cases}
$$

The 'keep or kill' hard thresholding operation is not the only reasonable way of estimating the coefficients. Recognizing that each coefficient $\tilde{w}_{j ; \eta}$ consists of both a signal portion and a noise portion, it might be desirable to attempt to isolate the signal contribution by removing the noisy part. This idea leads to the soft thresholding function as considered by D.L. DonOHO, I.M. Johnstone (1994)

$$
\delta_{\lambda}^{\text {soft }}(x)=\operatorname{sgn}(x) \max \{0,|x|-\lambda\}
$$

which can also be used in the identities (147) stated above. When soft thresholding is applied to a set of empirical coefficients, only coefficients greater than the threshold (in absolute value) are included, but their values are 'shrunk' toward zero by an amount equal to the threshold $\lambda$.

Summarizing all our results we finally obtain the following thresholding multiscale estimator

$$
\begin{aligned}
(\widehat{\Lambda F})_{J} & =\sum_{i=1}^{N_{J_{0}}} \delta_{k_{J_{0}}}\left(\tilde{a}_{i}^{N_{J_{0}}}\right) \Phi_{\rho_{J_{0}}}^{(2)}\left(\cdot \eta_{i}^{N_{J_{0}}}\right) \\
& +\sum_{j=J_{0}}^{J-1} \sum_{i=1}^{N_{j}} \delta_{k_{j}}\left(\tilde{a}_{i}^{N_{j}}\right) \Psi_{\rho_{j}}^{(2)}\left(\cdot \eta_{i}^{N_{j}}\right) .
\end{aligned}
$$

In doing so $(\widehat{\Lambda F})_{J}$ first is approximated by a thresholded $(\widetilde{\Lambda F})_{J_{0}}$, which represents the smooth components of the data. Then the coefficients at higher resolutions are thresholded, so that the noise is suppressed but the fine-scale details are included in the calculation. 


\section{Selective Multiscale Inversion of Pseudodiffer- ential Equations}

Finally our purpose is to develop a thresholded multiscale approximation method that allows a reconstruction of $\Lambda^{-1} G=F$ from error-affected 'data' $\tilde{G}=\widetilde{\Lambda F}$.

\subsection{The Operator Equation}

Consider a sequence $\left\{\Phi_{\rho_{j}}^{(2)}\right\}_{j \in \mathbb{Z}}$, with $\Phi_{\rho_{j}}^{(2)} \in \mathcal{L}^{2}[-1,+1]$ for all $j \in \mathbb{Z}$, constituting an approximate identity in $\mathcal{L}^{2}(\Omega)$ (in the sense of Section 1.3). Suppose that $\Lambda: \mathcal{L}^{2}(\Omega) \rightarrow \mathcal{L}^{2}(\Omega)$ is an (invariant) pseudodifferential operator with the following properties:

(i) for all integers $n \in \mathbb{N}_{0}$

$$
\Lambda^{\wedge}(n) \neq 0
$$

(ii)

$$
\sum_{n=0}^{\infty}\left(\Lambda^{\wedge}(n)\right)^{2}<\infty
$$

Then it is easily seen that $\Lambda$ represents an injective, bounded, compact operator with infinite dimensional range. The image $\operatorname{im}(\Lambda)$ of $\Lambda$ is equal to the Sobolev-like space $\Lambda\left(\mathcal{L}^{2}(\Omega)\right)=\mathcal{H}\left(\left\{\left(\Lambda^{\wedge}(n)\right)^{-1}\right\} ; \Omega\right) \subset \mathcal{L}^{2}(\Omega)$ (for notational details the reader is referred to W. FREEDEN et al. (1998)). Hence, it is a well-known fact (see, for example, A.K. Louis (1989)) that $\Lambda^{-1}$ is not bounded on $\mathcal{L}^{2}(\Omega)$. Moreover, it is clear that the problem

$$
\Lambda \hat{F}_{J}=\hat{G}_{J}, \quad \hat{F}_{J} \in \mathcal{L}^{2}(\Omega),
$$

with

$$
\begin{aligned}
\hat{G}_{J} & =\sum_{i=1}^{N_{J_{0}}} \delta_{k_{J_{0}}}\left(\tilde{a}_{i}^{N_{J_{0}}}\right) \Phi_{\rho_{J_{0}}}^{(2)}\left(\cdot \eta_{i}^{N_{J_{0}}}\right) \\
& +\sum_{j=J_{0}}^{J-1} \sum_{i=1}^{N_{j}} \delta_{k_{j}}\left(\tilde{a}_{i}^{N_{j}}\right) \Psi_{\rho_{j}}^{(2)}\left(\cdot \eta_{i}^{N_{j}}\right)
\end{aligned}
$$

is solvable if and only if $\hat{G}_{J}$ is a member of $\operatorname{im}(\Lambda)$, i.e. $\hat{G}_{J}$ satisfies the 
spectral condition

$$
\sum_{n=0}^{\infty} \sum_{k=1}^{2 n+1}\left(\frac{\left(\hat{G}_{J}\right)^{\wedge}(n, k)}{\Lambda^{\wedge}(n)}\right)^{2}<\infty .
$$

In our approach the last estimate, of course, can be understood as a restriction on the family $\left\{\Phi_{\rho_{j}}^{(2)}\right\}_{j \in \mathbb{Z}}$ constituting an approximate identity in $\mathcal{L}^{2}(\Omega)$. More explicitly, the operator equation (153) is uniquely solvable from the 'data' (154) by the function

$$
\begin{aligned}
\hat{F}_{J} & =\sum_{i=1}^{N_{J_{0}}} \delta_{k_{J_{0}}}\left(\tilde{a}_{i}^{N_{J_{0}}}\right) \Lambda^{-1}\left(\Phi_{\rho_{J_{0}}}^{(2)}\left(\cdot \eta_{i}^{N_{J_{0}}}\right)\right) \\
& +\sum_{j=J_{0}}^{J-1} \sum_{i=1}^{N_{j}} \delta_{k_{j}}\left(\tilde{a}_{i}^{N_{j}}\right) \Lambda^{-1}\left(\Psi_{\rho_{j}}^{(2)}\left(\cdot \eta_{i}^{N_{j}}\right)\right)
\end{aligned}
$$

if and only if

$$
\sum_{n=0}^{\infty} \frac{2 n+1}{4 \pi}\left(\frac{\left(\Phi_{\rho_{j}}^{\wedge}(n)\right)^{2}}{\Lambda^{\wedge}(n)}\right)^{2}<\infty
$$

for $j=J_{0}, \ldots, J$. In consequence, all bandlimited families $\left\{\Phi_{\rho_{j}}\right\}_{j \in \mathbb{Z}}$ can be used within the solution process.

As significant examples of operator equations we finally mention two important problems in modern physical geodesy.

\subsection{The Stokes' Problem}

Denote by $\mathcal{L}_{1}^{2}(\Omega)$ the space of functions $F \in \mathcal{L}^{2}(\Omega)$ satisfying $F^{\wedge}(0,1)=0$ and $F^{\wedge}(1,1)=F^{\wedge}(1,2)=F^{\wedge}(1,3)=0$. The problem of determining the anomalous potential on the earth's surface from given gravity anomalies $\hat{G}_{J}$ of the form (154) is provided by the Stokes, operator $\Lambda: \mathcal{L}_{1}^{2}(\Omega) \rightarrow$ $\mathcal{L}_{1}^{2}(\Omega)$ given by $\Lambda^{\wedge}(n)=(n-1)^{-1}, n=2,3, \ldots$ Obviously, the operator $\Lambda$ fulfills the (canonically modified) properties (151) and (152). The anomalous potential $\hat{F}_{J}$ is uniquely determined by $(156)$ if and only if

$$
\sum_{n=2}^{\infty} \frac{2 n+1}{4 \pi}(n-1)^{2}\left(\Phi_{\rho_{j}}^{\wedge}(n)\right)^{2}<\infty
$$

for $j=J_{0}, \ldots, J-1$. But this means that in addition to bandlimited functions $\Phi_{\rho_{j}}, j=J_{0}, \ldots, J$, certain types of non-bandlimited kernel functions 
can be used within the computation, too. Examples (cf. Section 1.4.2) are the Abel-Poisson singular integral or the locally supported singular integral with $k \geq 1$. Another useful example is the Tikhonov-Philips kernel defined by

$$
\Phi_{\rho_{j}}^{\wedge}(n)=\frac{\left(\Lambda^{\wedge}(n)\right)^{2}}{\left(\Lambda^{\wedge}(n)\right)^{2}+\rho_{j}^{2}\left(n+\frac{1}{2}\right)^{2}}, n=2,3, \ldots .
$$

\subsection{The Satellite-Gravity-Gradiometry Problem}

The problem of determining the gravitational potential $\hat{F}_{J}$ on the 'earth's sphere' (with radius $R$ ) from second order radial derivatives at the 'orbital sphere' (with radius $\gamma>R$ ) (for more mathematical details see W. FREEDEN (1999)) can be formulated by an operator equation $\Lambda \hat{F}_{J}=\hat{G}_{J}$, where the symbol $\left\{\Lambda^{\wedge}(n)\right\}_{n \in \mathbb{N}_{0}}$ is given by

$$
\Lambda^{\wedge}(n)=\left(\frac{R}{\gamma}\right)^{n} \frac{(n+1)(n+2)}{\gamma^{2}}, \quad n=0,1, \ldots .
$$

Obviously, the properties (151) and (152) are satisfied. The solvability condition reads as follows:

$$
\sum_{n=0}^{\infty} \frac{2 n+1}{4 \pi}\left(\frac{\gamma}{R}\right)^{2 n}\left(\frac{\gamma^{2}}{(n+1)(n+2)}\right)^{2}\left(\Phi_{\rho_{j}}^{\wedge}(n)\right)^{2}<\infty .
$$

Of course, all bandlimited kernel functions or (bandlimited) truncations of non-bandlimited kernel functions fulfill the last condition. But it is also possible to choose the Tikhonov-Philips-kernel in the aforementioned form, whereas the Abel-Poisson Singular Integral merely satisfies the solvability condition if and only if $0<\log \frac{\gamma}{R} \leq \rho_{j}$, which is not fulfilled for sufficiently large $\mathrm{j}$, such that this kernel is not appropriate in that case.

In particular for bandlimited white noise of the form (cf. R. RummeL (1997))

we find

$$
K(\xi \cdot \eta)=\frac{\sigma^{2}}{4 \pi} P_{0}(\xi \cdot \eta), \quad(\xi, \eta) \in \Omega \times \Omega,
$$

$$
k_{j}=\frac{2 \sqrt{\pi}}{N_{j}} \sigma\left(\Phi_{\rho_{j}}^{\wedge}(0)\right)^{2}, \quad j=J_{0}, \ldots, J-1
$$

This yields

$$
\hat{F}_{J}=\sum_{i=0}^{N_{J_{0}}} \delta_{\frac{2 \sqrt{\pi}}{N_{J_{0}}} \sigma\left(\Phi_{\rho_{J_{0}}}^{\wedge}(0)\right)^{2}}\left(\tilde{a}_{i}^{N_{J_{0}}}\right) \Lambda^{-1}\left(\Phi_{\rho_{J_{0}}}^{(2)}\left(\cdot \eta_{i}^{N_{J_{0}}}\right)\right)
$$




$$
+\sum_{j=J_{0}}^{J-1} \sum_{i=0}^{N_{J_{0}}} \delta_{\frac{2 \sqrt{\pi}}{N_{j}} \sigma\left(\Phi_{\rho_{j}}(0)\right)^{2}}\left(\tilde{a}_{i}^{N_{j}}\right) \Lambda^{-1}\left(\Psi_{\rho_{j}}^{(2)}\left(\cdot \eta_{i}^{N_{j}}\right)\right)
$$

as selective approximation of the satellite-gravity-gradiometry problem.

Acknowledgement: The third author acknowledges the financial support by the 'Graduiertenkolleg Technomathematik' of the University of Kaiserslautern.

\section{References}

Chui, C.K. (1992) An Introduction to Wavelets. Academic Press, San Diego, London.

Cui, J., Freeden, W., Witte, B. (1992) Gleichmäßige Approximation mittels sphärischer Finite-Elemente und ihre Anwendung auf die Geodäsie. Z. f. Vermessungswes., ZfV, 117, 266-278.

Donoho, D.L., Johnstone, I.M. (1994) Ideal Spatial Adaptation by Wavelet Shrinkage. Biometrika, 81, 425-455.

Donoho, D.L., Johnstone, I.M. (1995) Adapting to Unknown Smoothness Via Wavelet Shrinkage. J. Amer. Statistical Association, 90, 1200-1224.

Freeden, W. (1999): Multiscale Modelling of Spaceborne Geodata, B.G. Teubner, Stuttgart, Leipzig

Freeden, W., Gervens, T., Schreiner, M. (1998) Constructive Approximation on the Sphere (With Applications to Geomathematics). Oxford Science Publications, Clarendon.

Freeden, W., Hesse, K., (2000) Locally Supported Difference Wavelets on the Sphere. AGTM-Report 225, Laboratory of Technomathematics, Geomathematics Group, University of Kaiserslautern.

Freeden, W., Michel, V. (1999) Constructive Approximation and Numerical Methods in Geodetic Research Today - An Attempt at a Categorization Based on an Uncertainty Principle. J. of Geodesy, 73, 452-465.

Grafarend, E.W. (1982) Six Lectures on Geodesy and Global Geodynamics. Mitt. Geod. Inst. TU Graz, Folge 41 (H. Moritz, H. Sünkel, eds.), 531-685. 
Heiskanen, W.A., Moritz, H. (1967) Physical Geodesy. W.H. Freeman and Company.

LEBEDEw N.N. (1973) Spezielle Funktionen und ihre Anwendung. BIWissenschaftsverlag, Mannheim, Wien, Zürich.

Louis, A.K. (1989) Inverse und schlecht gestellte Probleme. B.G. Teubner, Stuttgart, Leipzig.

Louis, A.K., Maass, P., Rieder, A. (1994) Wavelets. B.G. Teubner Studienbücher, Stuttgart, Leipzig.

MeissL, P.A. (1971) A Study of Covariance Functions Related to the Earth's Disturbing Potential. The Ohio State University, Department of Geodetic Science and Surveying, Columbus, OSU Report No. 152.

Michel, V. (1999) A Multiscale Method for the Gravimetry Problem - Theoretical and Numerical Aspects of Harmonic and Anharmonic Modelling. Doctoral Thesis, University of Kaiserslautern, Geomathematics Group, Shaker Verlag, Aachen.

Moritz, H. (1980) Advanced Physical Geodesy. Herbert Wichmann Verlag, Karlsruhe.

MÜLlER, C. (1966) Spherical Harmonics. Lecture Notes in Mathematics, 17, Springer, Berlin, Heidelberg.

Ogden, R.T. (1997) Essential Wavelets for Statistical Applications and Data Analysis. Birkhäuser, Boston, Basel, Berlin.

Rummel, R. (1992) Fysische Geodesie I,II. Collegediktaat, Faculteit der Geodesie, Technische Universiteit Delft, Delft.

Rummel, R. (1997) Spherical Spectral Properties of the Earth's Gravitational Potential and its First and Second Derivatives. In: Lecture Notes in Earth Sciences, 65, 359-404.

Sansò, F., Rummel, R. (eds.) (1997) Geodetic Boundary Value Problems in View of the One Centimeter Geoid. Lecture Notes in Earth Sciences, 65, Springer, Berlin, Heidelberg.

Svensson, S.L. (1983) Pseudodifferential Operators-A New Approach to the Boundary Value Problems of Physical Geodesy. Manuscr. Geod., 8, 1-40. 
Weaver, J.B., Yansun, X., Healy, D.M.JR., Cromwell L.D. (1991): Filtering Noise From Images With Wavelet Transforms. Magnetic Resonance in Medicine, 24 288-295. 